\title{
Design of Generalized Analog Network Coding for a Multiple-Access Relay Channel
}

\author{
Sha Wei, Jun Li, Member, IEEE, Wen Chen, Senior Member, IEEE, Lizhong Zheng, and Hang Su
}

\begin{abstract}
In this paper, we propose a generalized analog network coding (GANC) scheme for a non-orthogonal multiple-access relay channel (MARC), where two sources transmit their information simultaneously to the destination with the help of a relay. In the GANC scheme, the relay receives interfered signals from the two sources and generates signals to be transmitted with a relay function. We focus on the design of the optimal relay function to achieve the minimum pair-wise error probability (PEP) of the system. Specifically, we first covert the relay function optimization problem to a transformation matrix (TM) design problem by presenting the received complex signals as signal matrices composed of real and imaginary parts. Then, we propose an optimization criteria, i.e., maximizing the minimal squared Euclidean distance (MMSED), to improve the PEP performance, since the PEP is determined by the Euclidean distance of the received constellation at destination. Next, we prove that the MMSED can be equivalently converted to a convex problem by introducing an intermediate matrix. We solve this convex problem by using the Lagrangian method and obtain the closed-form expression of the optimal TM. We further improve the PEP performance by optimizing transmission power of the two sources. Simulation results show that the proposed GANC scheme has a better PEP performance compared to other alternative schemes.
\end{abstract}

Index Terms-Relay function, multiple access relay channel, error probability, Euclidean distance.

\section{INTRODUCTION}

$\mathbf{C}$ OOPERATIVE communication has been investigated for decades as an efficient method to improve the reliability of wireless networks by exploiting spatial diversity via intermediate relay nodes [1], [2]. Network coding (NC), on the other hand, originated from wire-line networks [3], [4], has been

Manuscript received May 4, 2014; revised August 25, 2014 and October 14, 2014; accepted November 19, 2014. Date of publication December 2, 2014; date of current version January 14, 2015. This work was supported by the National 973 Project 2012CB316106, by NSF China under Grant 61161130529 and Grant 61328101, by National Huge Project under Grant 2015ZX03001028, by STCSM Science and Technology Innovation Program 13510711200, and by SEU National Key Lab on Mobile Communications under Grant 2013D11. The associate editor coordinating the review of this paper and approving it for publication was J. Yuan.

S. Wei, and H. Su are with the Department of Electronic Engineering, Shanghai Jiaotong University, Shanghai 200240, China (e-mail: venessa724@ sjtu.edu.cn; Hmilyanjohn@sjtu.edu.cn).

$\mathrm{J} . \mathrm{Li}$ is with the School of Electrical and Information Engineering, The University of Sydney, Sydney, N.S.W. 2006, Australia (e-mail: jun.li1@ sydney. edu.au).

W. Chen is with the Department of Electronic Engineering, Shanghai Jiaotong University, Shanghai 200240, China, and also with The School of Electronic Engineering and Automation, Guilin University of Electronic Technology, Guangxi 541004, China (e-mail: wenchen@ @jtu.edu.cn).

L. Zheng is with the Department of Electrical Engineering and Computer Sciences, Massachusetts Institute of Technology, Cambridge, MA 02139 USA (e-mail: lizhong@mit.edu).

Color versions of one or more of the figures in this paper are available online at http://ieeexplore.ieee.org.

Digital Object Identifier 10.1109/TCOMM.2014.2376954 recently applied to wireless channels to enhance the network throughput [5]. With the implementations of diversity techniques and network coding at the relay nodes, it is anticipated that wireless networks can achieve reliable and high throughput communications.

The NC based relaying schemes in multi-user systems can be broadly divided into two categories, namely, decode-andforward (DF) based and amplify-and-forward (AF) based $\mathrm{NC}$ schemes. In the DF based NC schemes, relay nodes decode received bit sequences and combine them together by using XOR or superposition coding before forwarding. In [6]-[11], the authors consider orthogonal multiple-access relay channels (MARC) without considering multi-user interference, leading to a low spectrum efficiency. In [12], a non-orthogonal MARC is studied with additive white Gaussian noise (AWGN) channels. Therefore, channel fading and diversity gain are not considered. In [13], the authors study both the orthogonal and non-orthogonal MARC. Particularly in the non-orthogonal MARC, the authors address the issue of the multi-user interference, and propose a network coded selective-and-forward relaying scheme to achieve full diversity gain by dropping erroneous messages.

Another important DF based NC scheme is physical-layer network coding (PNC) [14], which is mainly focussed on the two-way relay channel and achieves higher capacity than straight-forward network coding. Recently, the idea of PNC is extended to a $K$-user MARC system [15], where all the source nodes and the relay node transmit at the second phase. To achieve full diversity, the authors propose a decoder based on the possibility of error events at relay node, and form the network coding mapping with Latin Hypercube. Combining the PNC with structured lattice codes, the authors [16] propose a compute-and-forward (CPF) scheme in a multi-user Gaussian relay channel, where the relay node exploits the interference structure of the wireless channel and tries to decode the noisy received signal into integer linear combinations of codewords. Without particular assumption on the structure of the nested lattice codes, authors in [17] follow and generalize the framework of initial CPF scheme [16] using an algebraic approach. They give the sufficient condition for lattice partitions to have a vector space structure, based on which they generalize the lattice code construction and develop encoding and decoding methods. The authors in [18] derive the optimal dither method for lattice network coding (LNC) over GF(4), where they also construct parameters of LNC from convolutional, BCH, and Reed-Solomon codes.

In the AF based NC schemes [14], [19], [20], relay nodes simply amplify the received superimposed signals without decoding 
and forward them to the destination. A typical AF based $\mathrm{NC}$ scheme is analog network coding (ANC) [20]. In a two-way relay channels (TWRC), an ANC scheme is proposed [20], in which the relay amplifies and forwards interfered signals, and the terminals detect the signals from the counterpart by removing their own information. The ANC scheme is essentially a form of linear self-interference cancelation with the use of a priori known information. In [21], the authors derive both the outage probability and diversity-multiplexing tradeoff in a multiple source-destination pairs system, where the relay performs the ANC. In [22], the relay deploys a cooperative and multicast ANC scheme so that two destinations can use the interfered signals to recover the desired packet. The authors analyze such protocol in terms of the achieved rate and present an algorithm to recover signals from the overlapped transmissions. In [23], the authors propose a joint relay-selection and ANC using differential modulations in a bidirectional multi-relay systems. The ANC scheme and the CPF scheme are essentially physicallayer network coding (PNC) scheme, which exploit the interference structure of the wireless channel at relay node. As classified in [24], analog network coding is a PNC implemented in infinite-field, while the CPF scheme is a PNC implemented in finite-field. In infinite-field PNC, the relay node match the two sources-to-relay channels to reduce estimation errors; while finite-field PNC generates less redundant information at relay.

Compared with the DF based NC schemes, the ANC is more attractive for its simplicity. However, the ANC is inferior to the DF based NC schemes in terms of coding gain. This is due to the amplification of the received noise at the relay node, leading to the received constellation points at destination too close to each other. Properly designing a relay function in the ANC to enlarge the minimal distance of the constellation points can significantly improve the error performance, while keeping the simplicity of the ANC.

In this paper, we propose a generalized ANC (GANC) scheme for a non-orthogonal MARC over fading channels to achieve the minimization of the pairwise error probability (PEP). We particularly focus on the optimal relay function design to minimize the PEP. Firstly, we present a generalized form of the relay function, which can adjust the transmission power and phase on the received signals from the two sources. To simplify the optimization and analysis, we covert the relay function to a transformation matrix by presenting the received complex signals as signal matrices composed of real and imaginary parts. As the transformation matrix is in a generalized form for the GANC, it turns out to be an identity matrix for the conventional ANC.

Then we consider to optimize the transformation matrix to minimize the PEP of the system. By defining a symbol pair as two symbols from the two sources, we develop the expression of PEP based on the received constellation at the destination. Since the system PEP is determined by the Euclidean distance of the received constellation at destination, we propose an optimization criteria, i.e., maximizing the minimal squared Euclidean distance (MMSED), to improve the PEP performance.

Next, we prove that the MMSED problem can be equivalently converted to a convex optimization problem by in-

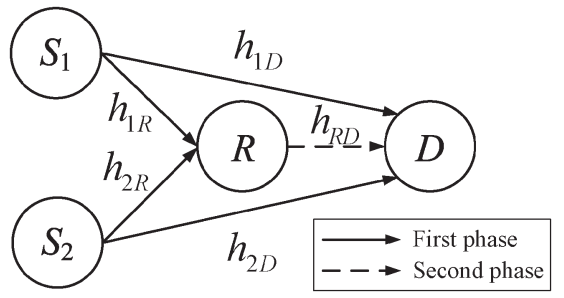

Fig. 1. The non-orthogonal MARC system with two sources, one relay and one destination.

troducing an intermediate matrix. As such, we can develop the optimal transformation matrix to achieve the solution of the MMSED by using the Lagrangian method. Specifically, we can convert the original MMSED optimization problem to a convex quadratical linear programming problem by (1) replacing the objective function in the MMSED with an affine function, and (2) defining an intermediate matrix to be optimized, which is the multiplication of the relay-to-destination channel matrix and the transformation matrix. In this case, we utilize the Lagrangian method and derive the optimal solution by discussing the possible values of Lagrangian multipliers. Then we can obtain the optimal transformation matrix based on the optimized intermediate matrix and the relay-to-destination channel matrix. With the optimized transformation matrix, we optimize transmission power of the two sources to further enhance the PEP performance. Simulation results show that the proposed GANC scheme has a better PEP performance comparing to other alternative schemes in both 4-QAM and 16QAM modulations.

The rest of this paper is organized as follows. We first describe the system model and propose the GANC relay function in Section II. Then we formulate the PEP minimization problem and derive the optimal relay function in Section III. Next, we investigate the practical aspect of the optimal relay function by discussing the feedback overhead in Section IV. The sources transmission power optimization problem is formulated and solved in Section V. Simulation results are summarized in Section VI, and conclusions are given in Section VII.

The notations used in this paper are as follows. Superscript $(\cdot)^{T}$ denotes transpose. Furthermore, $|x|$ denotes the norm of the complex number $x,\|\mathbf{x}\|_{2}^{2}$ denotes the squared Euclidean norm of a complex vector $\mathbf{x}$, and $\|\mathbf{X}\|_{F}^{2}$ denotes the Frobenius norm of a complex matrix $\mathbf{X}$.

\section{System Model}

Consider a two-source single-relay multiple-access relaying system, where two sources $\mathcal{S}_{1}$ and $\mathcal{S}_{2}$ transmit their information to the common destination $\mathcal{D}$ with the assistance of a halfduplex relay $\mathcal{R}$, shown in Fig. 1. Each transmission period is divided into two transmission phases. In a symbol time slot of the first transmission phase, the two sources simultaneously broadcast their symbols $x_{1}$ and $x_{2}$ to both the destination and the relay. In a symbol time slot of the second transmission phase, the two sources keep silent, while the relay processes the received signals and forwards the amplified symbol $x_{\mathscr{R}}$ to the destination. At the end of the second phase, the destination decodes the two sources' information based on the received signals. 
Let us define $x_{a}$ as one of an $M$-QAM symbol transmitted by $S_{a}$ for $a=1,2$, which consists of the in phase $x_{a I}$ and quadrature $x_{a Q}$ as $x_{a}=x_{a I}+\sqrt{-1} x_{a Q}$ with $\mathcal{E}\left(x_{a} x_{a}^{*}\right)=E_{a}$. Here, $E_{a}$ denotes the transmission power of $S_{a}$. In addition, the signal $x_{a b}$ for $b=I, Q$ is obtained as $x_{a b}=\mathcal{M}\left(s_{a b}\right)$, where $\mathcal{M}(\cdot)$ denotes a $\sqrt{M}$-PAM constellation mapper, and $s_{a b} \in\{0,1, \cdots, \sqrt{M}-1\}$ indicate the equally probable information corresponding to the in phase and quadrature of $x_{a}$, respectively. Then, $x_{a b}$ is calculated as

$$
x_{a b}=\frac{2 s_{a b}-(\sqrt{M}-1)}{\sqrt{2(M-1) / 3}} .
$$

Denote the $M$-QAM modulation set by $\Omega$. We assume that all the signals are transmitted in the same frequency band. The channel between any two nodes $v$ and $w, v \in\{1,2, \mathcal{R}\}, w \in$ $\{\mathcal{R}, \mathcal{D}\}$, and $v \neq w$, is denoted by $h_{v w}$ with a subscript indicating the nodes under consideration. We assume that $h_{v w}$ for all $v$ and $w$ are Rayleigh distributed with a mean zero and variance $\gamma_{v w}$. We consider slow fading channels in our system, i.e., the channel coefficients are constant during a transmission period, while they change independently from one transmission period to another. This assumption is practical in each subcarrier of an OFDM sytem.

Based on the aforementioned system settings and assumptions, the received signals at the relay and destination in a symbol time slot of the first transmission phase are

$$
\begin{gathered}
y_{\mathcal{R}}=\sqrt{E_{1}} h_{1 \mathcal{R}} x_{1}+\sqrt{E_{2}} h_{2 \mathcal{R}} x_{2}+n_{\mathcal{R}}, \\
y_{1}=\sqrt{E_{1}} h_{1 \mathcal{D}} x_{1}+\sqrt{E_{2}} h_{2 \mathcal{D}} x_{2}+n_{1}
\end{gathered}
$$

respectively, where $n_{\mathcal{R}}$ and $n_{1}$ are complex additive white Gaussian noise (AWGN) samples at the relay and destination with a zero mean and variance $\sigma^{2} / 2$ per dimension, respectively.

After receiving the interfered signal $y_{\mathcal{R}}$ from the two sources, the relay node applies a function $f(\cdot)$ on $y_{\mathcal{R}}$. The signal to be transmitted from the relay node can be written as

$$
x_{\mathcal{R}}=f\left(y_{\mathcal{R}}\right),
$$

where the specific definition of $f(\cdot)$ function will be introduced later. We assume that the maximum transmission power at relay node is $E_{\mathcal{R}}$, which yields $\left|x_{\mathcal{R}}\right|^{2} \leq E_{\mathscr{R}}$.

In a symbol time slot of the second transmission phase, the received signal at the destination can be expressed as

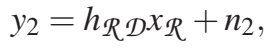

where $n_{2}$ is a complex AWGN sample with a zero mean and variance $\sigma^{2} / 2$ per dimension.
In (2)-(4), we have modeled the MARC system in the complex field. To simplify the optimization and analysis, the complex signals can be modeled in the real domain with matrices representations after some manipulations. In particular, by splitting the real and imaginary parts, the received signals $y_{\mathcal{R}}$ and $y_{1}$ in (2) can be represented as in (5), shown at the bottom of the page, where $\mathbf{x}=\left[\sqrt{E_{1}} x_{1}, \sqrt{E_{2}} x_{2}\right]^{T}$. According to the definitions in (5), we have

$$
\begin{aligned}
\mathbf{y}_{\mathcal{R}} & =\mathbf{I}_{2} \mathbf{H}_{\mathcal{R}} \mathfrak{R}\{\mathbf{x}\}+\mathbf{I}_{2}^{\prime} \mathbf{H}_{\mathcal{R}} \mathfrak{I}\{\mathbf{x}\}+\mathbf{n}_{\mathcal{R}}, \\
\mathbf{y}_{1} & =\mathbf{I}_{2} \mathbf{H}_{1} \Re\{\mathbf{x}\}+\mathbf{I}_{2}^{\prime} \mathbf{H}_{1} \mathfrak{I}\{\mathbf{x}\}+\mathbf{n}_{1} .
\end{aligned}
$$

Correspondingly, the relay function $f\left(y_{\mathcal{R}}\right)$ on the signal $y_{\mathcal{R}}$ can be represented by the multiplication of a two-dimensional real transformation matrix $\mathbf{R}$ and the real vector $\mathbf{y}_{\mathcal{R}}$, i.e.,

$$
\mathbf{x}_{\mathcal{R}}=\mathbf{R y}_{\mathcal{R}},
$$

where the $2 \times 1$ real vector $\mathbf{x}_{\mathscr{R}}=\left[\mathfrak{R}\left\{x_{\mathcal{R}}\right\}, \mathfrak{I}\left\{x_{\mathcal{R}}\right\}\right]^{T}$ is the real and imaginary parts of $x_{\mathcal{R}}$, and

$$
\mathbf{R} \triangleq \beta \Theta, \quad \text { for } \quad \beta \in \mathbb{R}^{+}, \Theta \in \mathbb{R}^{2 \times 2}, \operatorname{Tr}\left(\Theta^{T} \Theta\right)=1,
$$

with the transmission power constraint that

$$
\beta^{2}=\frac{E_{\mathcal{R}}}{\operatorname{Tr}\left\{\mathbf{y}_{\mathcal{R}} \mathbf{y}_{\mathcal{R}}^{T}\right\}} .
$$

From (8), we can see that the transformation matrix $\mathbf{R}$ has two parts. The parameter $\beta$ serves as the power normalization factor similar to that in the conventional ANC schemes. The matrix $\Theta$ can be viewed as the adjustment of the power and phase on the received signal $\mathbf{y}_{\mathcal{R}}$, which can be optimized to improve the PEP performance. Denote matrix $\mathbf{R}$ by its elements as $\mathbf{R}=$ $\left[R_{11}, R_{12} ; R_{21}, R_{22}\right]$. Then the $f(\cdot)$ function can be expressed as

$$
\begin{aligned}
f\left(y_{\mathcal{R}}\right)=\left(R_{11} \Re\left\{y_{\mathcal{R}}\right\}\right. & \left.+R_{12} \mathfrak{I}\left\{y_{\mathcal{R}}\right\}\right) \\
& +\sqrt{-1}\left(R_{21} \Re\left\{y_{\mathcal{R}}\right\}+R_{22} \mathfrak{I}\left\{y_{\mathcal{R}}\right\}\right) .
\end{aligned}
$$

We will discuss the optimal transformation matrix $\mathbf{R}$ to minimize the PEP later.

In the second transmission phase, the received signal $y_{2}$ at the destination can be expressed in the form of the real vector

$$
\mathbf{y}_{2}=\mathbf{H}_{2} \mathbf{x}_{\mathcal{R}}+\mathbf{n}_{2},
$$

where $\mathbf{y}_{2}=\left[\mathfrak{R}\left\{y_{2}\right\}, \mathfrak{I}\left\{y_{2}\right\}\right]^{T}, \mathbf{x}_{\mathscr{R}}=\left[\mathfrak{R}\left\{x_{\mathfrak{R}}\right\}, \mathfrak{I}\left\{x_{\mathscr{R}}\right\}\right]^{T}, \mathbf{n}_{2}=$ $\left[\Re\left\{n_{2}\right\}, \mathfrak{I}\left\{n_{2}\right\}\right]^{T}$, and

$$
\mathbf{H}_{2}=\left[\begin{array}{cc}
\mathfrak{R}\left\{h_{\mathcal{R D}}\right\} & -\mathfrak{I}\left\{h_{\mathcal{R} D}\right\} \\
\mathfrak{I}\left\{h_{\mathcal{R} \mathcal{D}}\right\} & \mathfrak{R}\left\{h_{\mathcal{R} \mathcal{D}}\right\}
\end{array}\right] .
$$

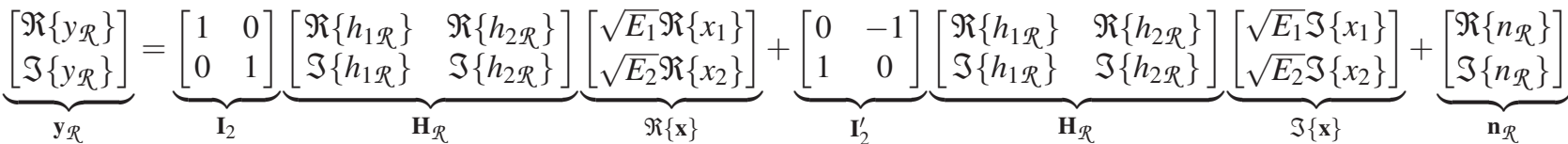

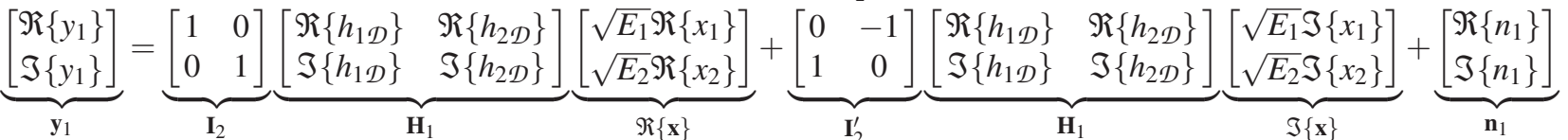


To simplify the analysis, we assume that the noise variance $\sigma^{2}$ equals one without a loss of generality. The destination performs maximum-likelihood detection (MLD) [25] to jointly decode the two source symbols based on the observations $\mathbf{y}_{1}$ and $\mathbf{y}_{2}$ is given in (13), shown at the bottom of the page, where $(\hat{*})$ denotes the detected symbol, and $(\tilde{*})$ denotes the trial symbol used in the hypothesis-detection problem. Since $\operatorname{Tr}\left(\mathbf{R}^{T} \mathbf{R}\right)=\beta^{2}$, the non-numeric part of denominator of the second term on the right side of the equation in (13) can be reformulated to

$$
\operatorname{Tr}\left\{\mathbf{H}_{2} \mathbf{R R}^{T} \mathbf{H}_{2}^{T}\right\}=\beta^{2} \operatorname{Tr}\left\{\mathbf{H}_{2} \mathbf{H}_{2}^{T}\right\} .
$$

For BPSK modulation, the MLD is simplified to

$$
\hat{\mathbf{x}}=\underset{\widehat{\mathbf{x}} \in \Omega}{\arg \min }\left(\left\|\mathbf{y}_{1}-\mathbf{H}_{1} \widetilde{\mathbf{x}}\right\|_{2}^{2}+\frac{\left\|\mathbf{y}_{2}-\mathbf{H}_{2} \mathbf{R} \mathbf{H}_{\mathcal{R}} \widetilde{\mathbf{x}}\right\|_{2}^{2}}{\beta^{2} \operatorname{Tr}\left\{\mathbf{H}_{2} \mathbf{H}_{2}^{T}\right\} / 2+1}\right) .
$$

Since the ML detection of the proposed GANC scheme in (13) is working symbol by symbol, there are $12 M^{2}$ number of second-order matrix multiplication and $7 M^{2}$ number of second-order matrix addition involved in each iteration. For the ANC scheme, there are $11 M^{2}$ number of second-order matrix multiplication and $7 M^{2}$ number of second-order matrix addition involved in each iteration, which does not calculate the $\mathbf{H}_{2} \mathbf{R}$ comparing with the proposed GANC scheme. Although the computational complexity of the proposed GANC scheme is slightly larger than the ANC scheme, the difference is on the factor of the complexity and the order is the same. And the GANC scheme has better coding gain than the ANC scheme as shown in Section IV.

\section{Optimization ON THE TRANSFORMATION MATRIX}

In this section, we discuss how to optimize the transformation matrix $\mathbf{R}$ to minimize the PEP. Firstly, we present the definition of the PEP. Then we propose a maximizing the minimal squared Euclidean distance (MMSED) criteria to improve the PEP performance, since system PEP is determined by the Euclidean distance of the received constellation at destination. Next, we convert the MMSED to a convex optimization problem since the MMSED problem is non-convex. We implement the Lagrangian method to solve the convex optimization and demonstrate the closed-form solution of the transformation matrix $\mathbf{R}$.

\section{A. Matrix Optimization}

The system PEP is defined as the probability that a transmitted symbol pair $\mathbf{x}$ is mistaken by a different symbol $\hat{\mathbf{x}}$. Define $\mathbf{x}_{i}$ and $\mathbf{x}_{j}$ as two different realizations of $\mathbf{x}$. Since each entry of both vectors $\mathbf{x}_{i}$ and $\mathbf{x}_{j}$ has $M$ possible values for $M$-QAM modulation, we have $i, j \in\left\{1,2, \cdots, M^{2}\right\}, i \neq j$. Given a channel realization vector $\mathbf{h}=\left[h_{1 \mathcal{R}}, h_{2 \mathcal{R}}, h_{1 \mathcal{D}}, h_{2 \mathcal{D}}, h_{\mathcal{R} \mathcal{D}}\right]$. According to
(13), the general expression of the system PEP of mistaking $\mathbf{x}_{i}$ by $\mathbf{x}_{j}$ is given by

$$
\operatorname{Pr}\left(\mathbf{x}_{i} \rightarrow \mathbf{x}_{j} \mid \mathbf{h}\right)=Q\left(\sqrt{\frac{D_{i j}}{2}}\right)
$$

where the $Q(x)$ function is defined as

$$
Q(x)=\frac{1}{\sqrt{2 \pi}} \int_{x}^{\infty} \exp \left(-z^{2} / 2\right) d z
$$

and $D_{i j}$ is the Euclidean distance between $\mathbf{x}_{i}$ and $\mathbf{x}_{j}$ at the ML detector given in (13).

Now, we investigate the instantaneous destination constellation (IDC) for a given channel realization $\mathbf{h}$, which is fourdimensional. Specifically, the $\mathrm{X}$-axis of IDC is the real signal part of $\mathbf{y}_{1}$, i.e., $\sqrt{E_{1}} \Re\left\{h_{1} \mathcal{D}\right\} \Re\left\{x_{1}\right\}+\sqrt{E_{2}} \Re\left\{h_{2} \mathcal{D}\right\} \Re\left\{x_{2}\right\}-$ $\sqrt{E_{1}} \mathfrak{I}\left\{h_{1 \mathcal{D}}\right\} \mathfrak{I}\left\{x_{1}\right\}-\sqrt{E_{2}} \mathfrak{I}\left\{h_{2 \mathcal{D}}\right\} \mathfrak{I}\left\{x_{2}\right\}$, the Y-axis of IDC is the imaginary signal part of $\mathbf{y}_{1}$, i.e., $\sqrt{E_{1}} \mathfrak{R}\left\{h_{1}\right\} \mathfrak{D}\left\{x_{1}\right\}+$ $\sqrt{E_{2}} \mathfrak{I}\left\{h_{2 \mathcal{D}}\right\} \Re\left\{x_{2}\right\}+\sqrt{E_{1}} \mathfrak{R}\left\{h_{1 \mathcal{D}}\right\} \mathfrak{I}\left\{x_{1}\right\}+\sqrt{E_{2}} \mathfrak{\Re}\left\{h_{2} \mathcal{D}\right\} \mathfrak{I}\left\{x_{2}\right\}$, the Z-axis of IDC is the real signal part of $\mathbf{y}_{2}$, i.e., $\sqrt{E_{\mathcal{R}}}\left(\Re\left\{h_{\mathcal{R} \mathcal{D}}\right\} \mathfrak{R}\left\{x_{\mathcal{R}}\right\}-\mathfrak{I}\left\{h_{\mathcal{R} \mathcal{D}}\right\} \mathfrak{I}\left\{x_{\mathcal{R}}\right\}\right)$, and the T-axis of IDC is the imaginary signal part of $\mathbf{y}_{2}$, i.e., $\sqrt{E_{\mathcal{R}}}\left(\mathfrak{R}\left\{h_{\mathcal{R}} \mathcal{D}\right\} \mathfrak{I}\left\{x_{\mathcal{R}}\right\}+\right.$ $\left.\mathfrak{I}\left\{h_{\mathscr{R} D}\right\} \Re\left\{x_{\mathcal{R}}\right\}\right)$.

With the setup of IDC, the squared Euclidean distance between two arbitrary constellation points at destination is given by

$$
\begin{aligned}
D_{i j}=\left\|\mathbf{H}_{1} \mathbf{d}_{i j}^{\Re}+\mathbf{I}_{2}^{\prime} \mathbf{H}_{1} \mathbf{d}_{i j}^{\mathfrak{\Im}}\right\|_{2}^{2} & \\
& +\frac{\left\|\mathbf{H}_{2} \mathbf{R} \mathbf{H}_{\mathcal{R}} \mathbf{d}_{i j}^{\Re}+\mathbf{H}_{2} \mathbf{R} \mathbf{I}_{2}^{\prime} \mathbf{H}_{\mathcal{R}} \mathbf{d}_{i j}^{\mathfrak{\Im}}\right\|_{2}^{2}}{\beta^{2} \operatorname{Tr}\left\{\mathbf{H}_{2} \mathbf{H}_{2}^{T}\right\} / 2+1},
\end{aligned}
$$

where $\mathbf{d}_{i j}^{\Re}=\mathfrak{R}\left\{\mathbf{x}_{i}-\mathbf{x}_{j}\right\}$ and $\mathbf{d}_{i j}^{\mathfrak{I}}=\mathfrak{I}\left\{\mathbf{x}_{i}-\mathbf{x}_{j}\right\}$. Specially, for BPSK modulation, the squared Euclidean distance is briefly written as

$$
D_{i j}=\left\|\mathbf{H}_{1} \mathbf{d}_{i j}\right\|_{2}^{2}+\frac{\left\|\mathbf{H}_{2} \mathbf{R} \mathbf{H}_{\mathcal{R}} \mathbf{d}_{i j}\right\|_{2}^{2}}{\beta^{2} \operatorname{Tr}\left\{\mathbf{H}_{2} \mathbf{H}_{2}^{T}\right\} / 2+1},
$$

where $\mathbf{d}_{i j}=\mathbf{x}_{i}-\mathbf{x}_{j}$. For $M$-QAM modulation, there exists totally $M^{4}-M^{2}$ number of squared Euclidean distances $D_{i j}$ since the distances when $i=j$ are excluded. Define the minimum Euclidean distance by $D_{\min }=\min \left\{D_{12}, D_{13}, \cdots, D_{i j}, \cdots\right.$, $\left.D_{M^{2}\left(M^{2}-1\right)}\right\}$. To optimize the PEP based on the ML detection, the minimum distance $D_{\min }$ should be maximized. Although in [26] we have proposed a power adaptive network coding (PANC) scheme, where the optimal power levels are obtained also based on the maximizing the minimal Euclidean distance criteria, there are some major differences between the PANC scheme and the proposed GANC scheme in this paper. Firstly, in [26], the derivation of error performance and optimizations are based on the BPSK modulation, i.e., $x_{1}, x_{2}, x_{\mathcal{R}} \in\{ \pm 1\}$, while in this paper, the performance analysis and optimizations are applicable for any QAM modulations. Secondly, in [26],

$$
\hat{\mathbf{x}}=\underset{\widetilde{\mathbf{x}} \in \Omega}{\arg \min }\left(\left\|\mathbf{y}_{1}-\mathbf{H}_{1} \Re\{\widetilde{\mathbf{x}}\}-\mathbf{I}_{2}^{\prime} \mathbf{H}_{1} \mathfrak{I}\{\widetilde{\mathbf{x}}\}\right\|_{2}^{2}+\frac{\left\|\mathbf{y}_{2}-\mathbf{H}_{2} \mathbf{R}\left(\mathbf{H}_{\mathcal{R}} \Re\{\widetilde{\mathbf{x}}\}-\mathbf{I}_{2}^{\prime} \mathbf{H}_{\mathcal{R}} \mathfrak{I}\{\widetilde{\mathbf{x}}\}\right)\right\|_{2}^{2}}{\operatorname{Tr}\left\{\mathbf{H}_{2} \mathbf{R R}^{T} \mathbf{H}_{2}^{T}\right\} / 2+1}\right)
$$


the channel phases are pre-equalized for both the source-todestination multiple access channels (MAC) and the relayto-destination channel before each transmission, while in this paper, pre-equalization is not needed and we consider a more general system model.

In that we consider slow fading channel, i.e., the channel coefficients are constant within a transmission period, the transformation matrix $\mathbf{R}$ is optimized for each transmission period. With the expression of the squared Euclidean distance given by (18), the Euclidean distance optimization problem under the relay power constraint in (9) is formulated as

$$
\mathbf{R}^{*}=\arg \max _{\mathbf{R}} \min _{\left\{\mathbf{d}_{i j}^{\text {qi }}, \mathbf{d}_{i j}^{\Im}\right\}}\left\{D_{i j}\right\} \quad \text { s.t. } \quad \operatorname{Tr}\left(\mathbf{R} \mathbf{R}^{T}\right)=\beta^{2} .
$$

Introduce $D_{\min }$ as a variable and define an intermediate matrix $\Psi \triangleq \mathbf{H}_{2} \mathbf{R}$. Also, define $\varphi \triangleq \frac{1}{\beta^{2} \operatorname{Tr}\left\{\mathbf{H}_{2} \mathbf{H}_{2}^{T}\right\} / 2+1}$. After some manipulations, the Euclidean distance optimization problem in (20) can be reformulated as a maximization problem over $D_{\min }$ and $\Psi$ as

$$
\begin{array}{ll}
\max \quad D_{\min } & \\
\text { s.t. } \quad D_{i j}= & \left\|\mathbf{I}_{2} \mathbf{H}_{1} \mathbf{d}_{i j}^{\Re}+\mathbf{I}_{2}^{\prime} \mathbf{H}_{1} \mathbf{d}_{i j}^{\mathfrak{T}}\right\|_{2}^{2} \\
& +\varphi\left\|\Psi \mathbf{I}_{2} \mathbf{H}_{\mathcal{R}} \mathbf{d}_{i j}^{\Re}+\Psi \mathbf{I}_{2}^{\prime} \mathbf{H}_{\mathcal{R}} \mathbf{d}_{i j}^{\mathfrak{T}}\right\|_{2}^{2} \geq D_{\min }, \\
\operatorname{Tr}\left(\Psi \Psi^{T}\right)= & \frac{2(1-\varphi)}{\varphi} .
\end{array}
$$

Noticing that $\left\|\mathbf{I}_{2} \mathbf{H}_{1} \mathbf{d}_{i j}^{\Re}+\mathbf{I}_{2}^{\prime} \mathbf{H}_{1} \mathbf{d}_{i j}^{\mathfrak{\Im}}\right\|_{2}^{2}$ is independent with matrix $\Psi$, we define $D_{i j}^{\text {direct }} \triangleq\left\|\mathbf{I}_{2} \mathbf{H}_{1} \mathbf{d}_{i j}^{\mathfrak{R}}+\mathbf{I}_{2}^{\prime} \mathbf{H}_{1} \mathbf{d}_{i j}^{\mathfrak{S}}\right\|_{2}^{2}$ to simplify our expression. Note that there are totally $M^{4}-M^{2}$ possible values of $D_{i j}$ involved in the first constraint of (21).

Due to the fact that in (21) the objective function of the maximization problem is an affine function and the constraints are quadratic functions of $\Psi$, the optimization problem is a convex quadratically constrained linear programming problem (QCLP). We can adopt the Lagrangian Multiplier method to obtain the optimal $\Psi$. In particular, the Lagrange equation is given by

$$
\begin{aligned}
& \mathcal{L}\left(\Psi, D_{\min },\left\{\mu_{i j}\right\}, \lambda\right) \\
& =D_{\min }+\sum_{i=1}^{M^{2}} \sum_{j=1, j \neq i}^{M^{2}} \mu_{i j}\left(D_{i j}^{\text {direct }}+\varphi\left\|\Psi \mathbf{u}_{i j}\right\|_{2}^{2}-D_{\min }\right) \\
& \quad-\lambda\left(\operatorname{Tr}\left(\Psi \Psi^{T}\right)-\frac{2(1-\varphi)}{\varphi}\right),
\end{aligned}
$$

where $\mu_{i j} \geq 0$ for all $i, j$ are Lagrange multipliers and $\mathbf{u}_{i j} \triangleq \mathbf{I}_{2} \mathbf{H}_{\mathcal{R}} \mathbf{d}_{i j}^{\Re \mathfrak{R}}+\mathbf{I}_{2}^{\prime} \mathbf{H}_{\mathcal{R}} \mathbf{d}_{i j}^{\mathfrak{S}}$. Since $D_{\min }, D_{i j}^{\text {direct }}$ and $\varphi$ are constants with respect to $\Psi$, the partial derivatives of these three constants over $\Psi$ are equal to zero. In addition, according to the relationship between the norm and the trace of a matrix, we have $\left\|\Psi \mathbf{u}_{i j}\right\|_{2}^{2}=\operatorname{Tr}\left(\Psi \mathbf{u}_{i j} \mathbf{u}_{i j}^{T} \Psi^{T}\right)$. Based on the matrix theory, we have $\frac{\partial \operatorname{Tr}\left(\mathbf{M}_{1} \mathbf{M}_{2} \mathbf{M}_{1}^{T}\right)}{\partial \mathbf{M}_{1}}=\mathbf{M}_{1} \mathbf{M}_{2}^{T}+\mathbf{M}_{1} \mathbf{M}_{2}$ for two arbitrary matrix $\mathbf{M}_{1}$ and $\mathbf{M}_{2}$. Thus, the Karush-Kuhn-Tucker (KKT) conditions are given by

$$
\begin{aligned}
& \frac{\partial \mathcal{L}}{\partial \Psi}=\varphi \sum_{i=1}^{M^{2}} \sum_{j=1, j \neq i}^{M^{2}} \mu_{i j} \Psi \mathbf{u}_{i j} \mathbf{u}_{i j}^{T}-\lambda \Psi=0, \\
& \frac{\partial \mathcal{L}}{\partial D_{\min }}=1-\sum_{i=1}^{M^{2}} \sum_{j=1, j \neq i}^{M^{2}} \mu_{i j}=0 .
\end{aligned}
$$

The complementary slackness conditions could be written as

$$
\begin{aligned}
& \mu_{i j}\left(D_{i j}^{\text {direct }}+\varphi\left\|\Psi \mathbf{u}_{i j}\right\|_{2}^{2}-D_{\min }\right)=0, \\
& \lambda\left(\operatorname{Tr}\left(\Psi \Psi^{T}\right)-\frac{2(1-\varphi)}{\varphi}\right)=0 .
\end{aligned}
$$

In the following theorem, we present the expression of the optimal matrix $\Psi^{*}$.

Theorem 1: The optimal matrix $\Psi^{*}$ that maximizes $D_{\min }$ can be presented as

$$
\begin{aligned}
& \Psi^{*}=\arg \max \left\{D_{\min }\left(\Psi_{k, i j}\right)\right\}, \\
& \text { for } i, j \in\left\{1,2, \cdots, M^{2}\right\}, i \neq j, k=1,2
\end{aligned}
$$

where $\Psi_{k, i j}$ are given in (26a) and (26b), shown at the bottom of the page, and $\kappa_{i j, 1}$ and $\kappa_{i j, 2}$ for $\kappa_{i j, 1}, \kappa_{i j, 2} \in \mathbb{R}^{+}$are two slack variables, which should satisfy the constraint that

$$
\kappa_{i j, 1}^{2}+\kappa_{i j, 2}^{2}=\frac{2(1-\varphi)}{\varphi\left(1+\frac{u_{i j, 2}}{u_{i j, 1}}\right)^{2}} .
$$

Besides, $u_{i j, 1}$ and $u_{i j, 2}$ are entries of the vector $\mathbf{u}_{i j} \triangleq \mathbf{I}_{2} \mathbf{H}_{\mathcal{R}} \mathbf{d}_{i j}^{\Re}+$ $\mathbf{I}_{2}^{\prime} \mathbf{H}_{\mathcal{R}} \mathbf{d}_{i j}^{\mathfrak{I}} ; u_{11}^{\{i j\}}, u_{12}^{\{i j\}}, u_{21}^{\{i j\}}$, and $u_{22}^{\{i j\}}$ are entries of matrix $\mathbf{U}^{\{i j\}} \triangleq \mathbf{u}_{i j} \mathbf{u}_{i j}^{T}-\mathbf{u}_{v w} \mathbf{u}_{v w}^{T}$ with $\mathbf{u}_{v w} \triangleq \mathbf{I}_{2} \mathbf{H}_{\mathcal{R}} \mathbf{d}_{v w}^{\Re}+\mathbf{I}_{2}^{\prime} \mathbf{H}_{\mathcal{R}} \mathbf{d}_{v w}^{\mathfrak{I}}$.

$$
\begin{aligned}
& \Psi_{1, i j}=\left[\begin{array}{ll}
\kappa_{i j, 1} & \frac{u_{i j, 2}}{u_{i j, 1}} \kappa_{i j, 1} \\
\kappa_{i j, 2} & \frac{u_{i j, 2}}{u_{i j, 1}} \kappa_{i j, 2}
\end{array}\right] \\
& \Psi_{2, i j}=\left[\begin{array}{cc}
\cos \left(\frac{\pi}{4}\right) & -\sin \left(\frac{\pi}{4}\right) \\
\sin \left(\frac{\pi}{4}\right) & \cos \left(\frac{\pi}{4}\right)
\end{array}\right]
\end{aligned}
$$

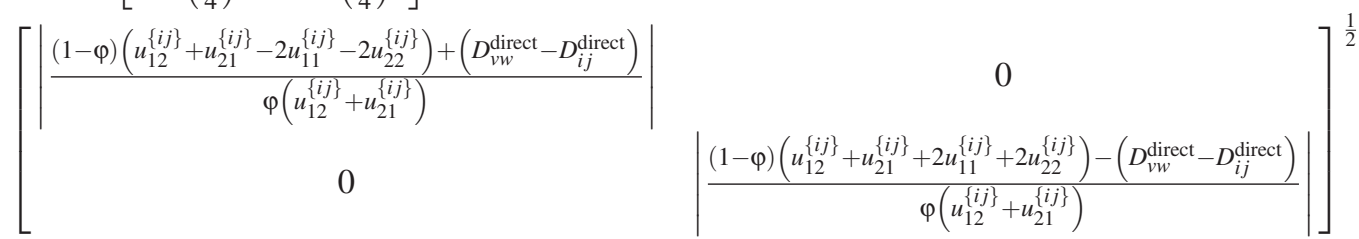




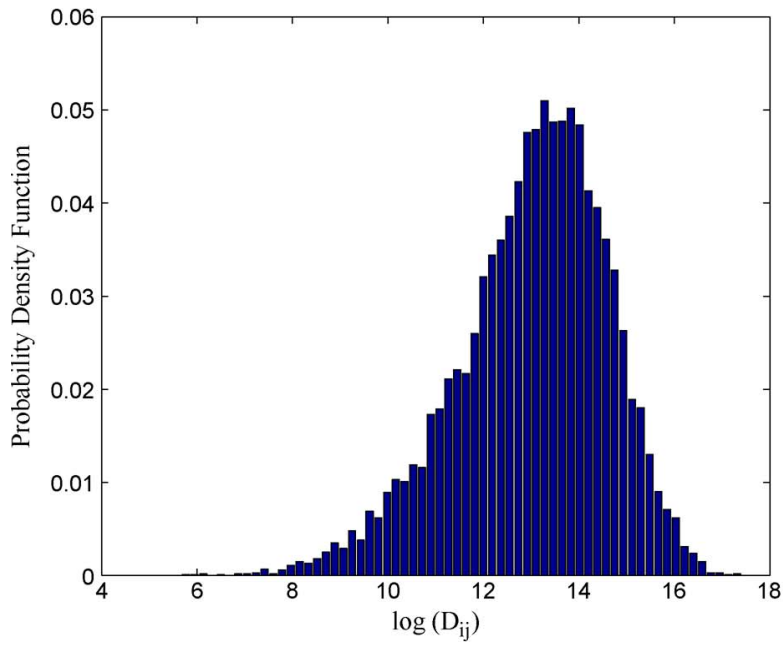

(a)

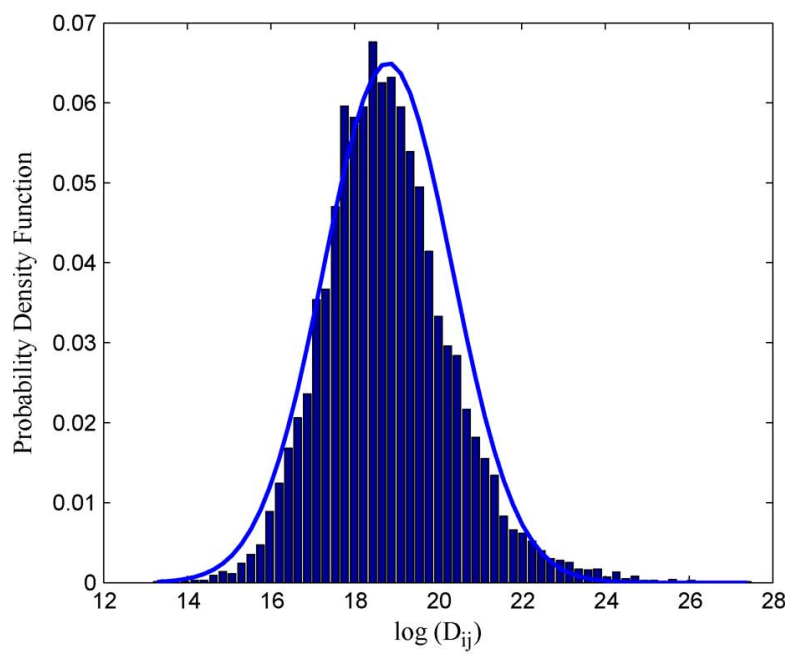

(b)

Fig. 2. Squared Euclidean distance of the GANC scheme and its scaled Gaussian fitting curve. (a) Squared Euclidean distance of the ANC scheme. (b) Squared Euclidean distance of the GANC scheme.

Proof: Please refer to Appendix A.

Based on the derivation result of the optimal $\Psi^{*}$, we can easily obtain the optimal transformation matrix $\mathbf{R}$ that maximizes $D_{\min }$, which is given as

$$
\mathbf{R}^{*}=\left(\mathbf{H}_{2}\right)^{-1} \Psi^{*} .
$$

\section{B. Performance Evaluations and Discussions}

In Fig. 2(a) and 2(b), we present the histogram of the minimum squared Euclidean distance for both the conventional ANC (refer to as ANC for simplicity) and GANC schemes, respectively. In particular, the sources transmission symbols are 4-QAM modulated. We generate 10000 realizations of the channel coefficients $h_{1 \mathcal{R}}, h_{2 \mathcal{R}}, h_{1 \mathcal{D}}, h_{2 \mathcal{D}}$, and $h_{\mathcal{R} \mathcal{D}}$. The system SNR is equal to $25 \mathrm{~dB}$. To facilitate the observation, the $\mathrm{X}$-label is chosen to be $\log \left(D_{i j}\right)$, which is quantized to 64 levels, and the Y-label is the probability density function of $\log \left(D_{i j}\right)$. We can see that comparing to the ANC scheme, the mean of the minimum squared Euclidean distance of the
GANC scheme is increasing from 11.53 to 20.33 , and the range of the distances is expanded from $[5.76,17.31]$ to $[13.28$, 27.39]. The closed-form expression for the distribution function of $\log \left(D_{i j}\right)$ is hard to derive due to the complicated form of optimal transformation matrix. Instead, we can implement a scaled Gaussian distribution $\frac{a_{1}}{\sqrt{2 \pi a_{3}}} \exp \left(-\frac{\left(x-a_{2}\right)^{2}}{2 a_{3}}\right)$ to fit the histgram, as shown in the solid line of Fig. 2(b), where $a_{1}$ is a positive scalar, $a_{2}$ is the mean of $\log \left(D_{i j}\right), a_{3}$ is the variance of $\log \left(D_{i j}\right)$, and the value range of $x$ is the same as $\log \left(D_{i j}\right)$. The simulation results reveal that the GANC greatly enlarges the minimum Euclidean distance of the received constellation comparing to the ANC.

Based on the discussion of the relationship between the transformation matrix and $D_{\min }$ in Appendix A, we learn that ANC scheme with $\mathbf{R}=\mathbf{I}_{2}$ is a special case of the proposed GANC scheme when $\operatorname{det}\left(\mathbf{F}_{i j}\right)=0$ and $u_{i j, 1}=u_{i j, 1}=2$, where $\mathbf{F}_{i j}$ is defined in (43). In other cases, the GANC scheme outperforms the ANC scheme in terms of PEP since it has a larger $D_{\min }$. This is also verified by the the simulation results of the probability density functions of $D_{\min }$ for both the GANC scheme and the ANC scheme in Fig. 2(a) and 2(b). Since the ANC scheme can achieve full diversity as proved in [25], our GANC scheme can also achieve full diversity.

From the expressions (26a), (26b), and (28), we can see that the optimal transformation matrix is determined by channel realizations, transmission power of two sources and relay, and the modulation that sources adopt. When only one squared Euclidean distance is comparably smaller than other distances, the intermediate variable $\Psi$ is determined by sources-to-relay channel only. While when two of the squared Euclidean distance are relatively smaller than other distances, the intermediate variable $\Psi$ is determined by both sources-to-relay channels and sources-to-destination channels. It is because the ML detection in (13) is a summation of both direct sources-todestination channels and cooperative sources-relay-destination channels.

When only one squared Euclidean distance is involved in optimization, since the direct channels are not a function of $\Psi$, the result of $\Psi$ is only related to the sources-to-relay channels. When two of the squared Euclidean distances are involved in optimization, we need to consider both the effects of direct channels and cooperative channels to balance the detection result. In addition, the optimal transformation matrix $\mathbf{R}^{*}$ in (28) is determined either by cooperative channels or all the communication channels corresponding to $\Psi^{*}$.

Comparing to the ANC scheme, where the $\mathbf{R}$ is equivalent to a two-dimensional identity matrix, the optimal transformation matrix not only amplifies the transmission power of the received signal, but also rotate the phase of the received signal by jointly considering the cooperative channels or all the communication channels. In this case, the design of the transformation matrix performs non-commutative transformation, i.e., both scaling and rotation, on the received signal to minimize the system PEP. In the proposed GANC scheme, the modulation level $M$ determines the complexity of the system, where $2\left(M^{4}-M^{2}\right)$ number of $D_{i j}$ are considered in the Lagrangian multiplier method. 


\section{Practical Consideration FOR MATRIX OPTIMIZATION}

To obtain the optimal transformation matrix $\mathbf{R}$, the optimization process can be implemented at either the destination or the relay, which requires different levels of feedback. Thus, the complexity of feedback varies in different circumstances.

\section{A. Optimization at Destination}

From the expressions (26a) and (26b), we can see that both solutions only contain two real numbers, i.e., $\kappa_{1}$ and $\kappa_{2}$ in (26a), and the two diagonal elements of the second matrix in (26b). Note that as the receiver, the relay node can easily obtain the instantaneous channel state information (CSI) of the sources-torelay channels, ${ }^{1}$ from which we can compute $u_{i j, 1}$ and $u_{i j, 2}$. The destination needs to know the global CSIs of the system, which is a common setting in other AF-based cooperative systems. After multiplying with $\mathbf{H}_{2}^{-1}$, the transformation matrix $\mathbf{R}$ is determined by two or four real numbers if $\Psi^{*}$ is obtained based on (26a) or (26a), respectively.

In practice, the relay does not need to know the CSIs of both sources-to-destination and relay-to-destination channels when the optimization process is executed at destination. It is because the destination can feedback the transformation matrix $\mathbf{R}$, which is calculated offline and contains two or four real numbers, to the relay. To be more practical, instead of transmitting the optimal $\mathbf{R}$ to the relay, the destination can quantize the real numbers in $\mathbf{R}$ to limited digits and feedback to the relay.

\section{B. Optimization at Relay}

When the optimization process is implemented at the relay, the feedback of different levels of instantaneous CSI affects the accuracy of the optimal $\mathbf{R}$. Firstly, we consider the case when the relay has the instantaneous CSI of the sources-to-relay channels, sources-to-destination channels and relay-to-destination channel. In this case, the destination needs to feedback three complex numbers or six real numbers, i.e., $h_{1 \mathcal{D}}, h_{2 \mathcal{D}}$, and $h_{\mathcal{R D}}$, to the relay. Although the cost of the feedback is high, we can obtain the accurate solution of the optimal $\mathbf{R}$.

Secondly, we consider the case when the relay has only the instantaneous CSI of the sources-to-relay channels, and the statistical CSI of both the sources-to-destination channels and relay-to-destination channel. As a receiver, the relay can easily obtain the instantaneous CSI of the sources-to-relay channels. In this case, the destination does not need to feedback any instantaneous CSI to the relay. In the following, we use the BPSK modulation as an example to demonstrate how to obtain $\mathbf{R}$ with the statistical CSI of the sources-to-destination channels and the relay-to-destination channel. From (19), we can see that the direct link related term $\left\|\mathbf{H}_{1} \mathbf{d}_{i j}\right\|_{2}^{2}$ is a function of $\mathbf{H}_{1}$. By applying the inequality $\|\mathbf{A B}\|_{2}^{2} \leq\|\mathbf{A}\|_{2}^{2}\|\mathbf{B}\|_{2}^{2}$, we have

$$
\left\|\mathbf{H}_{1} \mathbf{d}_{i j}\right\|_{2}^{2} \leq\left\|\mathbf{H}_{1}\right\|_{2}^{2}\left\|\mathbf{d}_{i j}\right\|_{2}^{2} .
$$

\footnotetext{
${ }^{1}$ To obtain the instantaneous CSI of sources-to-relay channels, we can adopt the ML estimator introduced in [27], [28] at relay node.
}

Since we only have the statistical information of $\left\|\mathbf{H}_{1}\right\|_{2}^{2}$, we use $\mathbb{E}\left\{\left\|\mathbf{H}_{1}\right\|_{2}^{2}\right\}$ to substitute $\left\|\mathbf{H}_{1}\right\|_{2}^{2}$ in (19), where $\mathbb{E}\{\cdot\}$ denotes the expectation. Consequently, the squared Euclidean distance of the direct link can be approximately calculated by

$$
\bar{D}_{i j}^{\text {direct }}=\mathbb{E}\left\{\left\|\mathbf{H}_{1}\right\|_{2}^{2}\right\}\left\|\mathbf{d}_{i j}\right\|_{2}^{2} .
$$

In addition, we use the statistical information $\mathbb{E}\left\{\left\|\mathbf{H}_{2}\right\|_{2}^{2}\right\}$ to substitute $\operatorname{Tr}\left\{\mathbf{H}_{2} \mathbf{H}_{2}^{T}\right\}$ in $\varphi$, that is

$$
\bar{\varphi}=\frac{1}{\beta^{2} \mathbb{E}\left\{\left\|\mathbf{H}_{2}\right\|_{2}^{2}\right\} / 2+1} .
$$

We can obtain $\Psi_{1, i j}$ and $\Psi_{2, i j}$ in (26a) and (26b) by substituting the $D_{i j}^{\text {direct }}$ and $\varphi$ with $\bar{D}_{i j}^{\text {direct }}$ and $\bar{\varphi}$ in (30) and (31), respectively. Denote the optimal matrix $\Psi^{*}$ derived with statistical CSI by $\bar{\Psi}^{*}$. For the matrix $\mathbf{R}$, we can use $\mathbb{E}\left\{\mathbf{H}_{2}^{-1}\right\}$ to substitute the value $\left(\mathbf{H}_{2}\right)^{-1}$ in (28), that is

$$
\overline{\mathbf{R}}^{*}=\mathbb{E}\left\{\mathbf{H}_{2}^{-1}\right\} \bar{\Psi}^{*} .
$$

\section{Sources Power Allocation With the TRANSFORMATION MATRIX DESIGN}

In this section, we optimize the sources transmission power $E_{a}$ for $a=1,2$ with the optimal matrix $\Psi^{*}$ derived in Section III. For the simplicity of expression, we use $\Psi$ instead of $\Psi^{*}$ in this Section. To assist the subsequent analysis, we decompose the distance vector $\mathbf{d}_{i j}^{\Re \mathfrak{R}}$ for $i, j \in\left\{1,2, \cdots, M^{2}\right\}, i \neq j$ into a multiplication of a sources transmission power related matrix and the real part of transmission signal related vector as

$$
\mathbf{d}_{i j}^{\Re} \triangleq \mathbf{E}_{\mathcal{S}} \widetilde{\mathbf{d}}_{i j}^{\Re}=\left[\begin{array}{cc}
\sqrt{E_{1}} & 0 \\
0 & \sqrt{E_{2}}
\end{array}\right]\left[\begin{array}{c}
\Re\left\{x_{1 i}-x_{2 i}\right\} \\
\Re\left\{x_{1 j}-x_{2 j}\right\}
\end{array}\right],
$$

where $x_{a i}$ and $x_{a j}$ are realizations of $x_{a}$ on the $M$-QAM constellation of source $a$. Likewise, we can decompose the distance vector $\mathbf{d}_{i j}^{\mathfrak{S}}$ by $\mathbf{d}_{i j}^{\mathfrak{I}}=\mathbf{E}_{\mathcal{S}} \widetilde{\mathbf{d}}_{i j}^{\mathfrak{S}}$ where $\widetilde{\mathbf{d}}_{i j}^{\mathfrak{I}}=\left[\mathfrak{I}\left\{x_{1 i}-x_{2 i}\right\}, \mathfrak{I}\left\{x_{1 j}-\right.\right.$ $\left.\left.x_{2 j}\right\}\right]^{T}$. Hence, the Euclidean distance optimization problem under the sum sources power constraint can be formulated as a maximization problem over $D_{\min }$ and $\mathbf{E}_{\mathcal{S}}$ as

$$
\begin{array}{ll} 
& \max D_{\min } \\
\text { s.t. } & \left\|\mathbf{H}_{1} \mathbf{E}_{\mathcal{S}} \widetilde{\mathbf{d}}_{i j}^{\Re}+\mathbf{I}_{2}^{\prime} \mathbf{H}_{1} \mathbf{E}_{\mathcal{S}} \widetilde{\mathbf{d}}_{i j}^{\mathfrak{S}}\right\|_{2}^{2} \\
& +\varphi\left\|\Psi \mathbf{H}_{\mathcal{R}} \mathbf{E}_{\mathcal{S}} \widetilde{\mathbf{d}}_{i j}^{\Re}+\Psi \mathbf{I}_{2^{\prime}} \mathbf{H}_{\mathcal{R}} \mathbf{E}_{\mathcal{S}} \widetilde{\mathbf{d}}_{i j}^{\mathfrak{S}}\right\|_{2}^{2} \geq D_{\min }, \\
& \left\|\mathbf{E}_{\mathcal{S}}\right\|_{2}^{2}=E_{\text {total }} .
\end{array}
$$

Due to the fact that the objective function of the maximization problem is an affine function and the constraints are quadratic functions of variable $\mathbf{E}_{\mathcal{S}}$ in (34), it is a convex quadratically constrained linear programming problem (QCLP). We can adopt the Lagrangian Multiplier method to obtain the optimal sources transmission power. In particular, the 
Lagrange equation is given in (35), shown at the bottom of the page. The Karush-Kuhn-Tucker (KKT) conditions are given by

$$
\begin{aligned}
& \frac{\partial \mathcal{L}}{\partial D_{\min }}=1-\sum_{i=1}^{M^{2}} \sum_{j=1, j \neq i}^{M^{2}} \mu_{i j}=0 \\
& \frac{\partial \mathcal{L}}{\partial \mathbf{E}_{\mathcal{S}}}=\sum_{i=1}^{M^{2}} \sum_{j=1, j \neq i}^{M^{2}} \\
& \times\left\{\mu _ { i j } \left[\left(\mathbf{H}_{1} \mathbf{H}_{1}^{T}+\varphi\left(\Psi \mathbf{H}_{\mathcal{R}}\right)\left(\Psi \mathbf{H}_{\mathcal{R}}\right)^{T}\right) \mathbf{E}_{\mathcal{S}} \widetilde{\mathbf{d}}_{i j}^{\Re}\left(\widetilde{\mathbf{d}}_{i j}^{\Re}\right)^{T}\right.\right. \\
& \quad+\left(\left(\mathbf{I}_{2}^{\prime} \mathbf{H}_{1}\right)\left(\mathbf{I}_{2}^{\prime} \mathbf{H}_{1}\right)^{T}+\varphi\left(\Psi \mathbf{I}_{2}^{\prime} \mathbf{H}_{\mathcal{R}}\right)\left(\Psi_{2}^{\prime} \mathbf{H}_{\mathcal{R}}\right)^{T}\right) \mathbf{E}_{\mathcal{S}} \widetilde{\mathbf{d}}_{i j}^{\mathfrak{S}}\left(\widetilde{\mathbf{d}}_{i j}^{\mathfrak{S}}\right)^{T} \\
& \left.\left.\quad+2\left(\left(\mathbf{I}_{2}^{\prime} \mathbf{H}_{1}\right)^{T} \mathbf{H}_{1}+\varphi\left(\Psi \mathbf{I}_{2}^{\prime} \mathbf{H}_{\mathcal{R}}\right)^{T} \Psi_{\mathcal{R}}\right) \mathbf{E}_{\mathcal{S}} \widetilde{\mathbf{d}}_{i j}^{\Re}\left(\widetilde{\mathbf{d}}_{i j}^{\mathfrak{I}}\right)^{T}\right]\right\} \\
& \quad-\lambda \mathbf{E}_{\mathcal{S}} \\
& =\mathbf{0}_{2 \times 2},
\end{aligned}
$$

The complementary slackness conditions could be written as

$$
\begin{aligned}
& \mu_{i j}\left(\left\|\mathbf{I}_{2} \mathbf{H}_{1} \mathbf{E}_{\mathcal{S}} \widetilde{\mathbf{d}}_{i j}^{\Re}+\mathbf{I}_{2}^{\prime} \mathbf{H}_{1} \mathbf{E}_{\mathcal{S}} \widetilde{\mathbf{d}}_{i j}^{\Im}\right\|_{2}^{2}\right. \\
& \left.\quad+\varphi\left\|\Psi \mathbf{H}_{\mathcal{R}} \mathbf{E}_{\mathcal{S}} \widetilde{\mathbf{d}}_{i j}^{\Re}+\Psi \mathbf{I}_{2}^{\prime} \mathbf{H}_{\mathcal{R}} \mathbf{E}_{\mathcal{S}} \widetilde{\mathbf{d}}_{i j}^{\mathfrak{S}}\right\|_{2}^{2}-D_{\min }\right)=0 \\
& \quad \lambda\left(\left\|\mathbf{E}_{\mathcal{S}}\right\|_{2}^{2}-E_{\text {total }}\right)=0 .
\end{aligned}
$$

Similar to the discussion in Section III, we maximize $D_{\min }$ by optimizing $\mathbf{E}_{\mathcal{S}}$. In the following theorem, we present the expression of the optimal sources transmission power.

Theorem 2: Given the total sources transmission power $E_{\text {total }}$, the optimal matrix $\mathbf{E}_{\mathcal{S}}$ that maximizes $D_{\min }$ can be presented as

$$
\begin{aligned}
& \mathbf{E}_{\mathcal{S}}^{*}=\arg \max \left\{D_{\min }\left(\mathbf{E}_{\mathcal{S}}^{k, i j}\right)\right\}, \\
& \text { for } \quad i, j \in\left\{1,2, \cdots, M^{2}\right\}, i \neq j, k=1,2,
\end{aligned}
$$

where $\mathbf{E}_{S}^{k, i j}$ are defined in (39a) and (39b), shown at the bottom of the page. $\eta_{3, i j}$ and $\eta_{4, i j}$ are defined in (53), and $\varphi_{1, m t}$ and $\varphi_{2, m t}$ are defined in (58) for $m t \in\{i j, v w\}$.

Proof: Please refer to Appendix B.
For the practical consideration, the destination needs to broadcast one real number to both sources for a channel realization, i.e., $\frac{\eta_{4, i j}}{\eta_{3, i j}}$ or $\frac{\varphi_{2, i j}-\varphi_{2, v w}}{\varphi_{1, i j}-\varphi_{1, v w}}$ for power allocation.

As we can see from the optimization process, the optimizations on both the transformation matrix and transmission power have closed forms. Each time when the channel changes, the optimization process of transformation matrix involves $12\left(M^{4}-M^{2}\right)$ number of second-order matrix multiplication and $4\left(M^{4}-M^{2}\right)$ number of second-order matrix addition, while the optimization process of sources transmission power involves $14\left(M^{4}-M^{2}\right)$ number of second-order matrix multiplication and $5\left(M^{4}-M^{2}\right)$ number of second-order matrix addition. Therefore, when the frame length is large, the overall complexity is relatively low. Apart from the optimization within each transmission period, the complexity also comes from the symbol-pair-based relay operation, which consists of a multiplication between a $2 \times 2$ optimization matrix and a $2 \times 1$ signal vector. While in the DF-based schemes, in which the symbols need to be recovered one-by-one, the detection complexity is based on the detector at the relay and the order of the modulations. For instance, if the relay chooses a maximum likelihood detector for DF scheme, the detection process at relay involves $2 M^{2}$ number of second-order matrix multiplication and $2 M^{2}$ number of second-order matrix addition each symbol pair. Denote the frame length by $L$. If $L>6\left(M^{2}-1\right)$, which is always hold for real-time wireless communication, the complexity of our GANC scheme is lower than the DF and other modified DF schemes, while with a better error performance as shown in Section VI.

There are mainly two advantages of the proposed GANC scheme comparing to the CPF scheme. Firstly, CPF scheme requires an $n$-dimensional [29] or $2 M L T$-dimensional lattice code [30] as its codebook, where $M$ is the number of antennas at transmitter, $L$ is the transmission slots and $T$ is the number of vector symbols, while GANC is applicable to any arbitrary $M$-QAM signals. Thus, without practical consideration of codebook design, the CPF scheme cannot be applied to the real

$$
\begin{aligned}
\mathcal{L}\left(D_{\min }, \mathbf{E}_{\mathcal{S}},\left\{\mu_{i j}\right\}, \lambda\right)=D_{\min }-\lambda( & \left.\left\|\mathbf{E}_{\mathcal{S}}\right\|_{2}^{2}-E_{\text {total }}\right)+\sum_{i=1}^{M^{2}} \sum_{j=1, j \neq i}^{M^{2}} \\
& \times\left\{\mu_{i j}\left(\left\|\mathbf{H}_{1} \mathbf{E}_{\mathcal{S}} \widetilde{\mathbf{d}}_{i j}^{\Re}+\mathbf{I}_{2}^{\prime} \mathbf{H}_{1} \mathbf{E}_{\mathcal{S}} \widetilde{\mathbf{d}}_{i j}^{\mathfrak{S}}\right\|_{2}^{2}+\varphi\left\|\Psi \mathbf{H}_{\mathcal{R}} \mathbf{E}_{\mathcal{S}} \widetilde{\mathbf{d}}_{i j}^{\Re}+\Psi \mathbf{I}_{2}^{\prime} \mathbf{H}_{\mathcal{R}} \mathbf{E}_{\mathcal{S}} \widetilde{\mathbf{d}}_{i j}^{\mathfrak{I}}\right\|_{2}^{2}-D_{\min }\right)\right\}
\end{aligned}
$$

$$
\begin{aligned}
\mathbf{E}_{\mathcal{S}}^{1, i j} & =\left[\begin{array}{cc}
\sqrt{E_{\text {total }} \sin \left(\arctan \left(-\frac{\eta_{4, i j}}{\eta_{3, i j}}\right)\right)} & 0 \\
0 & \sqrt{E_{\text {total }} \cos \left(\arctan \left(-\frac{\eta_{4, i j}}{\eta_{3, i j}}\right)\right)}
\end{array}\right] \\
\mathbf{E}_{\mathcal{S}}^{2, i j} & =\left[\begin{array}{cc}
\sqrt{E_{\text {total }} \sin \left(\arctan \left(-\frac{\varphi_{2, i j}-\varphi_{2, v w}}{\varphi_{1, i j}-\varphi_{1, v w}}\right)\right)} & 0 \\
0 & \sqrt{E_{\text {total }} \cos \left(\arctan \left(-\frac{\varphi_{2, i j}-\varphi_{2, v w}}{\varphi_{1, i j}-\varphi_{1, v w}}\right)\right)}
\end{array}\right]
\end{aligned}
$$


communication directly, while our GANC scheme is applicable to practical modulation system.

Secondly, GANC scheme does not detect received signal at relay node, hence has no detection error. Therefore, both the GANC scheme and conventional ANC scheme can achieve full diversity in the MARC system. However, for the CPF scheme, if the relay maps the received signal to a wrong lattice point, the error will propagate to the destination. Thus, like the decodeand-forward (DF) scheme, the CPF cannot achieve full diversity of the system due to the error propagation problem. Hence, our GANC scheme will have a better error performance than the $\mathrm{CPF}$ since it can achieve full diversity gain.

\section{Simulation}

In this section, we evaluate the performance of the proposed GANC scheme by simulations. We set the frame (or codeword) length as $l=10,000$. Hence, each transmission period is of length 20,000. As we consider quasi-static fading channels, the channel coefficients $h_{1 \mathcal{R}}, h_{2 \mathcal{R}}, h_{1 \mathcal{D}}, h_{2 \mathcal{D}}$, and $h_{\mathcal{R} \mathcal{D}}$ are constant in each transmission period, and change independently from one period to another. Throughout our simulations, we use the path loss model $\gamma_{v w}=d_{v w}^{-2}$, where $\gamma_{v w}$ is the channel gain, for $v \in\{1,2, \mathcal{R}\}, w \in\{\mathcal{R}, \mathcal{D}\}, v \neq w$. We assume that $E_{1}+E_{2}=$ $2 E_{\mathcal{R}}$, and the SNR in the simulation is defined as $\rho=E_{\mathcal{R}} / \sigma^{2}$.

We first focus on a symmetric scenario where the two sources have the same distance to the relay and the same distance to the destination. The distances between the sources and the destination are normalized as one, i.e., $d_{1 \mathcal{D}}=d_{2 \mathcal{D}}=1$. The relay is located between the sources and the destination. In our simulations, we consider three cases, namely, Case 1, Case 2, and Case 3, according to the three different locations of the relay. Specifically, in Case 1, we consider a strong sourceto-relay link scenario, where $d_{1 \mathcal{R}}=d_{2 \mathcal{R}}=0.3$, and $d_{\mathcal{R D}}=$ 0.7. In Case 2, we consider the symmetric scenario, where $d_{1 \mathcal{R}}=d_{2 \mathcal{R}}=0.5$, and $d_{\mathbb{R} \mathcal{D}}=0.5$. In Case 3, we consider a strong relay-to-destination scenario, where $d_{1} \mathcal{R}=d_{2 \mathcal{R}}=0.7$, and $d_{\mathcal{R} \mathcal{D}}=0.3$.

In each realization of nodes locations, we simulate the following seven schemes: (a) the PEP performance of the CXNC scheme [31], [32] in which the relay transmits an XORed signal to the destination in the second transmission phase, denoted by $C X N C$, (b) the PEP performance of the complex field network coding (CFNC) scheme [33] in which the relay transmits a network coded signal in complex field to the destination in the second transmission phase, denoted by $C F N C$, (c) the PEP performance of the physical-layer network coding (PNC) scheme [15] in which the relay transmits a network coded signal based on a modulo-4 Latin square, and all the sources and relay transmit in the second phase, denoted by $P N C$, (d) the PEP performance of the analog network coding scheme [20] in which the relay amplifies and forwards the powernormalized received signal to the destination in the second time phase, denoted by $A N C$, (e) the PEP performance of the proposed GANC scheme with the optimized transformation matrix (ITM) $\mathbf{R}$ (i.e., optimization at the destination) and equivalent sources transmission power, i.e., $E_{1}=E_{2}=E_{R}$, denoted by $G A N C, I T M$, (f) the PEP performance of the proposed GANC

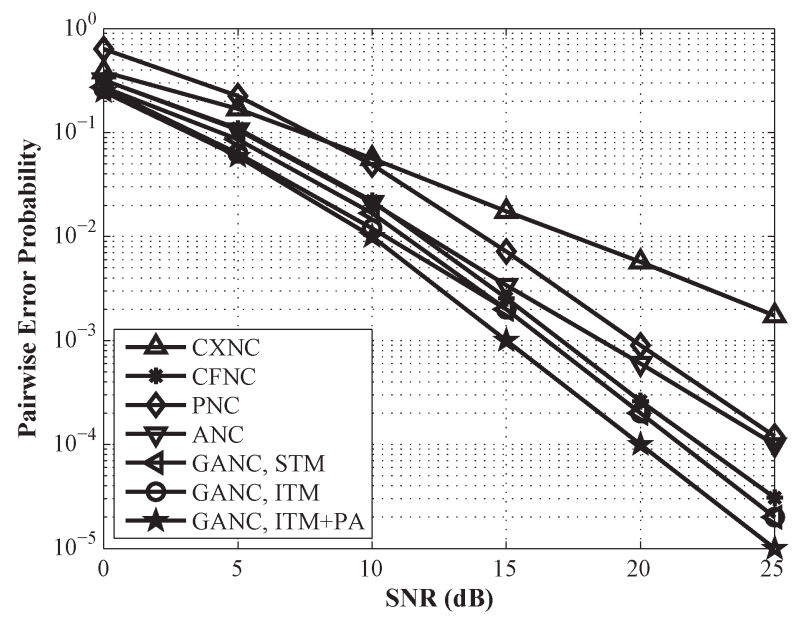

(a)

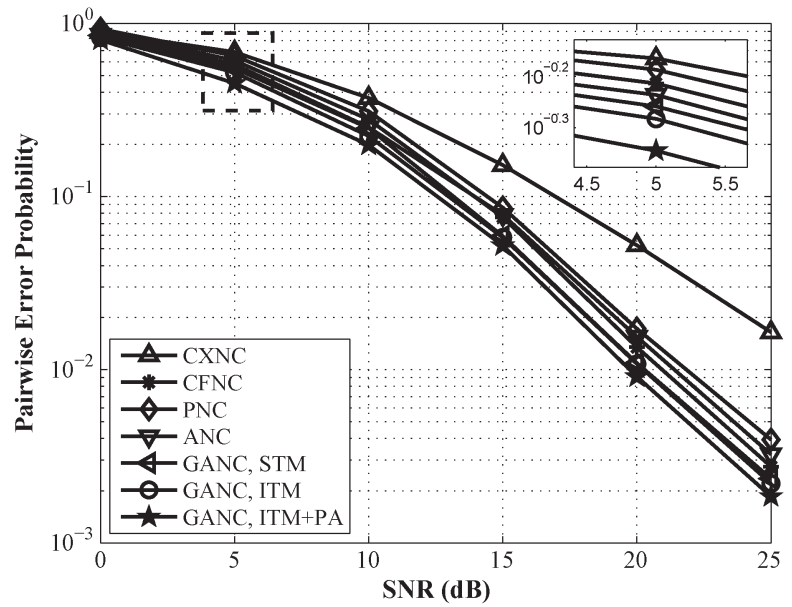

(b)

Fig. 3. Error performance for Case 1. (a). 4-QAM. (b). 16-QAM.

scheme with the statistical transformation matrix (STM) $\overline{\mathbf{R}}$ given in (32) (i.e., optimization at the relay) and $E_{1}=E_{2}=E_{\mathcal{R}}$, denoted by GANC, STM, and ( $\mathrm{g}$ ) the PEP performance of the proposed GANC scheme with the optimized transformation matrix $\mathbf{R}$ given in (28) and optimal sources power allocation (PA) given in Section IV by monte carlo simulation, denoted by $G A N C, I T M+P A$.

From Fig. 3(a) and (b) we can see that our proposed GANC schemes, i.e., GANC, ITM + PA, GANC, ITM and GANC, STM schemes, outperform $C X N C, C F N C, P N C$ and $A N C$ schemes in both 4-QAM and 16-QAM modulations. The reason why the PEP performance of the proposed GANC schemes are better than that of the alternative protocols is that the GANC schemes are optimized to minimize the PEP, while the rest of them are not, which cannot guarantee the optimality of the system PEP. The CFNC scheme is better than the ANC scheme since the sources-to-relay channels are relatively stronger than the relayto-destination channel. Specifically, for the $C F N C$ scheme, the reliability of the network coded symbol is high, which yields a higher confidence of the cooperative channels and a better overall error performance. While for the ANC scheme, the severe fading of the relay-to-destination channel greatly affect the error performance. The $P N C$ scheme can achieve full diversity for its special decoder design to mitigate error propagation, 


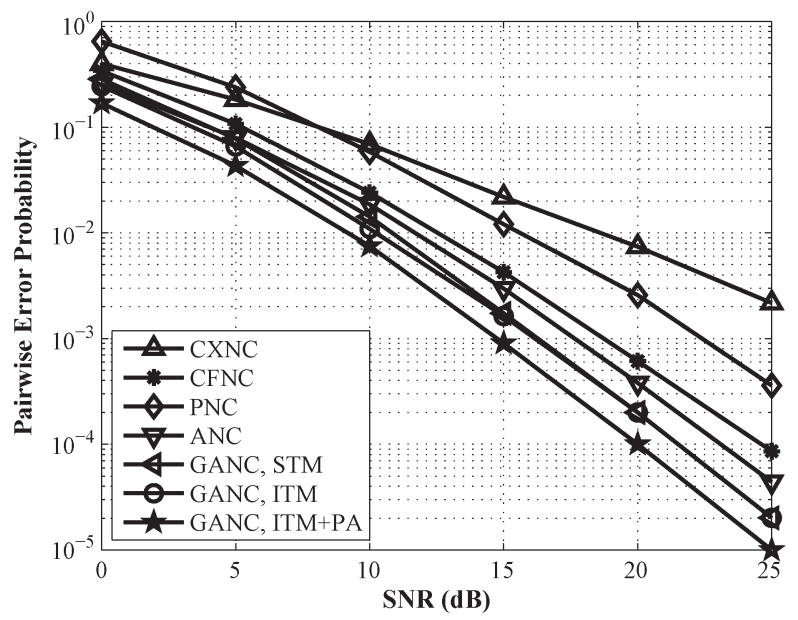

(a)

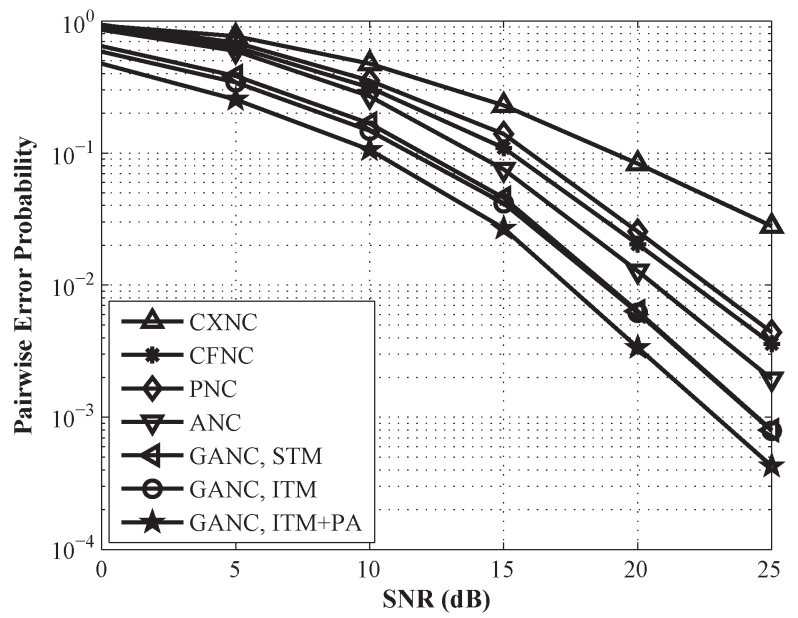

(b)

Fig. 4. Error performance for Case 2. (a) 4-QAM. (b) 16-QAM.

network coding operation drawn from a Latin Hypercube, and requirement of sources transmission in the second phase. The CXNC scheme performs worst.

Figs. 4(a), (b) and 5(a), (b) show the error performances of Case 2 and Case 3 for both 4-QAM and 16-QAM modulations, respectively. In both cases, our proposed GANC schemes can achieve the full diversity gain, and outperform the alternative protocols for both 4-QAM and 16-QAM modulations. As the relay being more close to destination, the $A N C$ scheme becomes better than the $C F N C$ scheme in terms of PEP performance. The $C X N C$ scheme still performs worst.

From the six figures, we can see that the average PEP exhibits decreasing coding gain as the constellation size increases. The $G A N C, I T M+P A$ scheme with optimized sources transmission power has better coding gain than the GANC,ITM scheme with equal transmission power. The GANC, ITM scheme outperforms $G A N C, S T M$ scheme since it gains benefit from feedback. The GANC schemes with both ITM and STM have better PEP performance than the alternative strategies. The $C X N C$ scheme can only achieve a diversity of one, due to the error propagation from the source-relay hop and the detection ambiguity of the multiple-to-one network coding mapping [26]. The $C F N C$ scheme with a precoding design at two sources and a complex field network coding design at relay can

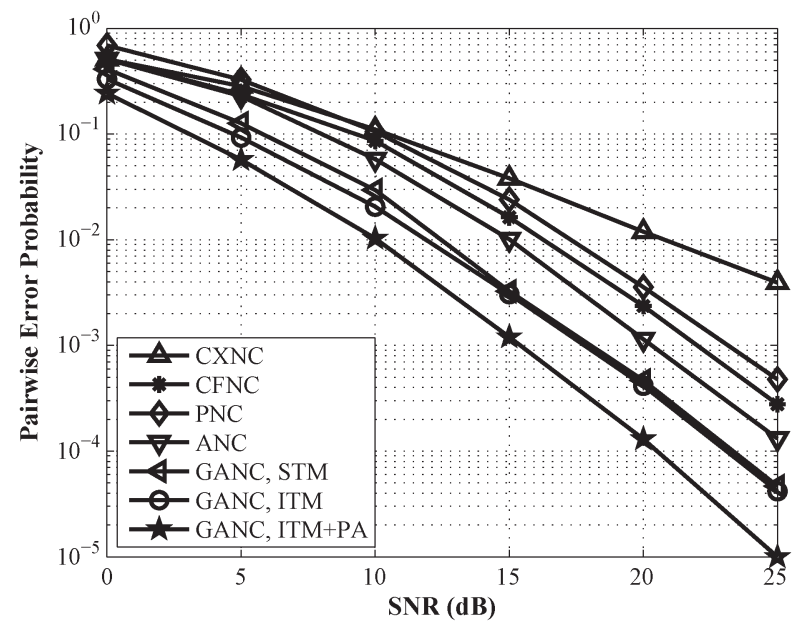

(a)

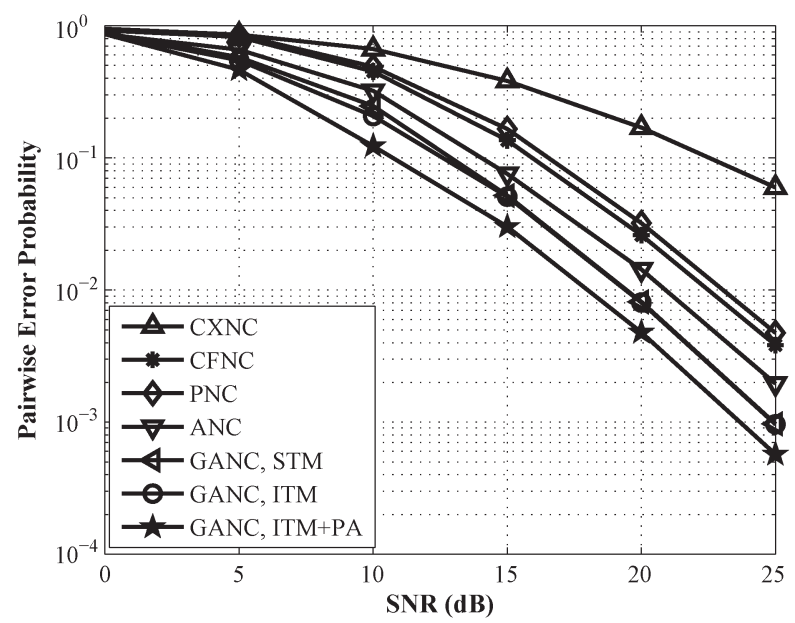

(b)

Fig. 5. Error performance for Case 3. (a) 4-QAM. (b) 16-QAM.

achieve full diversity. Note that, to achieve full diversity, the CFNC scheme adopts a LAR [33] at relay, which requires a feedback of instantaneous CSI of relay-to-destination channel. The $P N C$ scheme can also achieve full diversity. However, the coding gain of the PNC scheme is not sound, since the sources share a fixed transmission power in two phases and the error propagation mitigation technique works worse than link adaptive ratio (LAR) [33] in terms of PEP. The ANC scheme is proved to achieve full diversity at high SNRs in [25]. The proposed GANC scheme can achieve full diversity since it aims to minimize the PEP by maximizing the minimum Euclidean distance.

The performance gap between the benchmarks and the proposed GANC scheme become large when the relay is closer to the destination. As shown in [25], the quality of relay-todestination channel dominates the overall error performances of the $A N C$ scheme. Thus, the closer the relay to the destination, the better the error performance of the $A N C$ scheme, and vice versa. While for digital network coding schemes, i.e., the $C X N C$ scheme, the $C F N C$ scheme and the PNC scheme, the error propagation from the sources-to-relay hop greatly impact the overall error performance. In this case, the closer the relay to the sources, the better the error performance of the digital network coding schemes, and vice versa. 


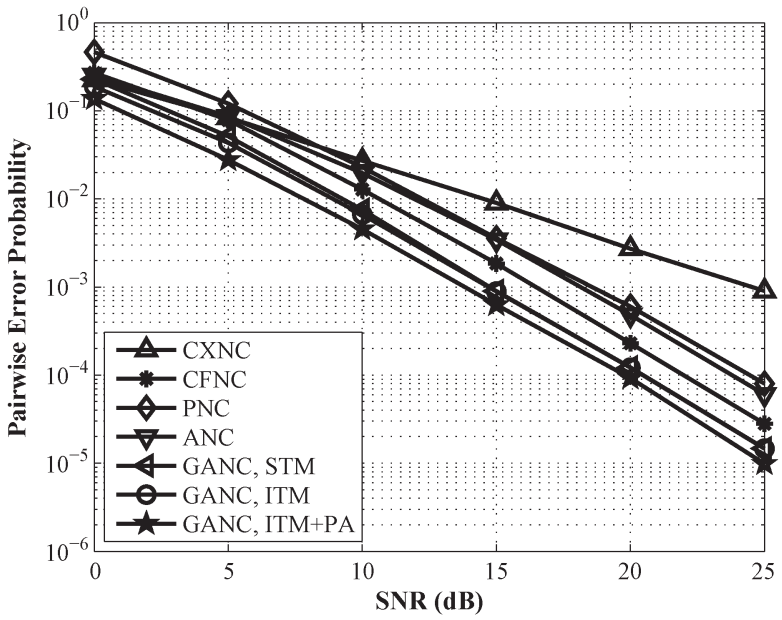

(a)

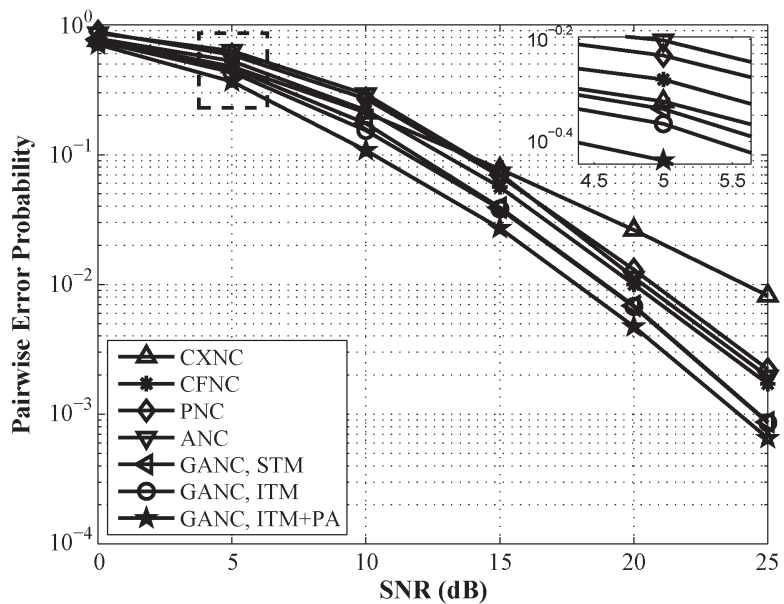

(b)

Fig. 6. Error performance for Case 4. (a) 4-QAM. (b) 16-QAM.

Furthermore, we consider two asymmetric scenarios, where the two sources are asymmetrically located. Specifically, we assume $d_{1 \mathcal{R}}=0.5, d_{2 \mathcal{R}}=0.2, d_{\mathcal{R} \mathcal{D}}=0.5, d_{1 \mathcal{D}}=1$, and $d_{2 \mathcal{D}}=$ 0.7 , denoted by Case $4 ; d_{1 \mathcal{R}}=0.7, d_{2 \mathcal{R}}=0.2, d_{\mathcal{R} D}=0.3$, $d_{1 \mathcal{D}}=1$, and $d_{2 \mathcal{D}}=0.5$, denoted by Case 5 . Figs. 6(a), (b) and 7(a), (b) show the error performances of Case 4 and Case 5 for both 4-QAM and 16-QAM modulations, respectively. From these figures, we can see that our proposed GANC schemes outperform the alternative schemes in terms of the PEP performance. The simulation results show that the performance comparisons of these protocols in asymmetric scenarios are consistent with those in the symmetric scenarios.

In Fig. 8, we also present the simulation results of the PANC scheme in [26] and the proposed GANC scheme based on BPSK modulation and channel pre-equalization for both the source-to-destination channel and the relay-to-destination channel. We can see that both the PANC scheme and the GANC scheme can achieve full diversity, and the coding gain of the proposed GANC scheme outperforms PANC scheme in [26].

\section{CONCLUSION}

In this paper, we propose a GANC scheme to minimize the system PEP for a non-orthogonal MARC. Firstly, we model the

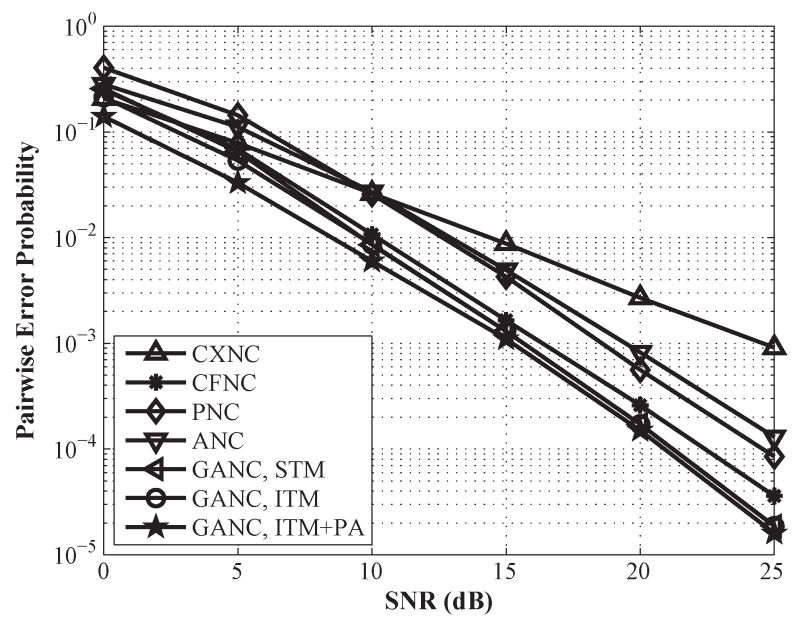

(a)

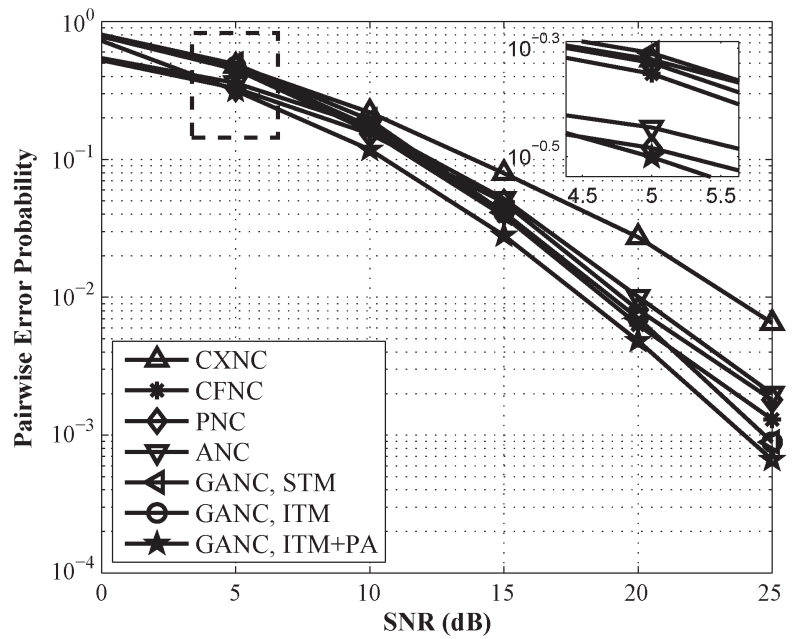

(b)

Fig. 7. Error performance for Case 5. (a) 4-QAM. (b) 16-QAM

transmission signals in both complex and real fields. Then, we propose an optimization criteria, i.e., maximizing the minimal squared Euclidean distance, to improve the PEP performance. In particular, we define the minimum squared Euclidean distance based on the maximum likelihood detection. Next, we formulate an optimization problem to maximize the minimum Euclidean distance under the relay transmission power constraint. Since the proposed optimization problem is convex, we utilize the Lagrangian method and obtain the closed-form expression of the transformation matrix by discussing the possible values of Lagrangian multipliers. Furthermore, we optimize the sources transmission power with the optimized transformation matrix. Simulation results show that the proposed GANC scheme has a better PEP performance compared to other alternative schemes in 4-QAM and higher order QAM modulations.

\section{APPENDIX}

\section{A. Proof of Theorem 1}

In the following, we derive the optimal matrix $\Psi$ by discussing the possible values of $D_{\min }$. For $M$-QAM modulation, there are generally two cases of values of $D_{\min }$ : (1) when only 


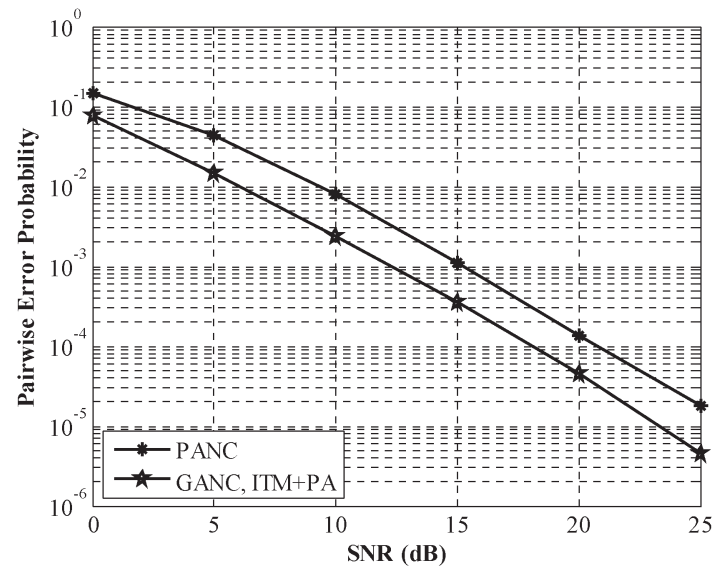

(a)

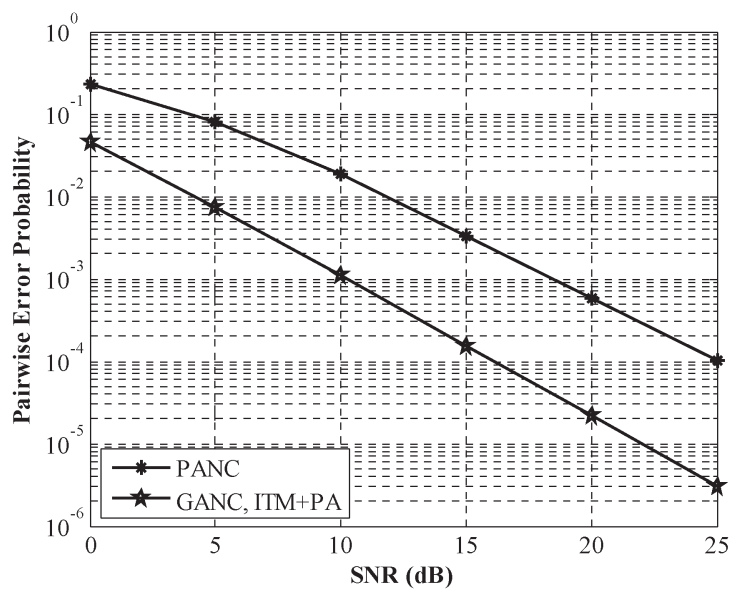

(c)

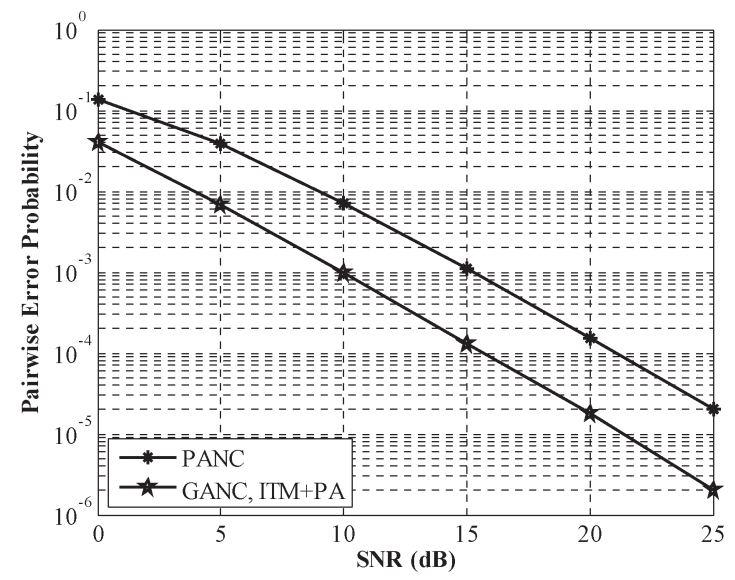

(b)

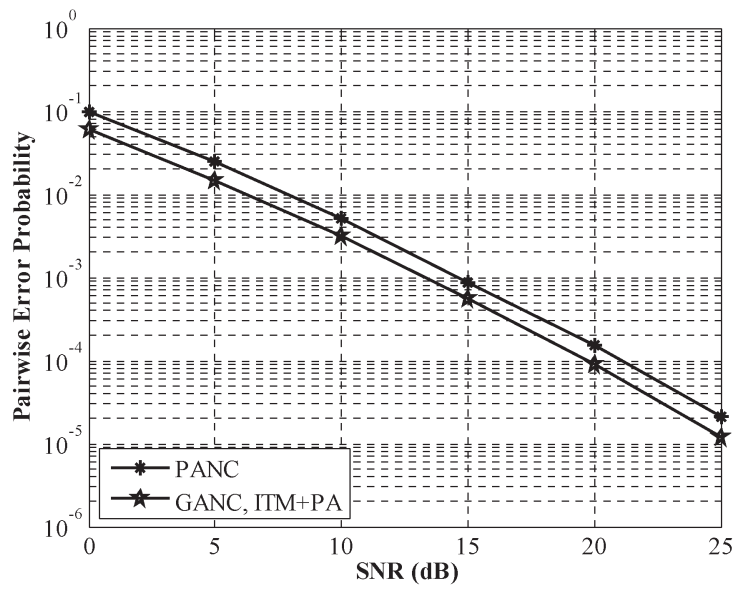

(d)

Fig. 8. Error performance of PANC scheme [26] and GANC scheme. (a) Case 1. (b) Case 2. (c) Case 3. (d) Case 4.

one squared Euclidean distance is smaller than other distances, i.e., $D_{\min }=D_{i j}$; and (2) when at least two squared Euclidean distances are equal to each other and also smaller than the rest of the distances, i.e., $D_{\min }=D_{i j}=D_{v w}$ for $i, j, v, w \in$ $\left\{1,2, \cdots, M^{2}\right\}, i \neq j, v \neq w$. Suppose that there are more than two squared Euclidean distances equalling to each other and also smaller than the rest of the distances, then each pair of equivalent distance will generate a matrix $\Psi$. If the derived matrix $\Psi$ s are the same, then one pair of equivalent distances is enough to obtain the optimal $\Psi^{*}$. Otherwise, there is no solution of matrix $\Psi$ which satisfies the condition that there are more than two squared Euclidean distances equalling to each other and also smaller than the rest of the distances. For instance, we assume $D_{\min }=D_{i j}=D_{v w}=D_{l z}$ for $i, j, v, w, l, z \in$ $\left\{1,2, \cdots, M^{2}\right\}, i \neq j, v \neq w, l \neq z$. Let $\Psi_{1}$ and $\Psi_{2}$ be the solutions of functions $D_{i j}=D_{v w}$ and $D_{i j}=D_{l z}$, respectively. If $\Psi_{1}=\Psi_{2}$, we can obtain the solution of function $D_{i j}=D_{v w}=D_{l z}$ by solving either the function $D_{i j}=D_{v w}$ or $D_{i j}=D_{l z}$. If $\Psi_{1} \neq \Psi_{2}$, there is no solution to the function $D_{\min }=D_{i j}=D_{v w}=D_{l z}$. From this example, we can see that discussing the solution of two equivalent distances is enough to obtain matrix $\Psi$. This conclusion can be easily extended to cases when more than three distances are involved. In this case, it is not necessary to discuss the situation when more than two squared Euclidean distances are equal to each other.
Then, the original optimization problem in (21) can be divided into $2\left(M^{4}-M^{2}\right)$ subproblems. Solving each subproblem will produce a matrix $\Psi^{\{k, i j\}}$ and its corresponding minimum squared Euclidean distance $D_{\min }^{\{k, i j\}}$, where the first superscript $k=1,2$ denotes the index of the cases and the second superscript pair $i j$ is corresponding to the involved Euclidean distance $D_{i j}$. Hence, the optimal matrix $\Psi^{*}$ is the one that corresponding to the maximal $\left\{D_{\min }^{\{k, i j\}}\right\}$.

In the first case when only one Euclidean distance is equal to $D_{\min }$, i.e., $\mu_{i j}>0,\left\{\mu_{v w}\right\}=0, \lambda \neq 0$ for $i, j, v, w \in$ $\left\{1,2, \cdots, M^{2}\right\}, i \neq j, v \neq w$, from the complementary slackness conditions in (24), there is only one Lagrangian multiplier $\mu_{i j}>0$, i.e.,

$$
D_{\min }=D_{i j}=D_{i j}^{\text {direct }}+\varphi\left\|\Psi^{\{1, i j\}} \mathbf{u}_{i j}\right\|_{2}^{2},
$$

where $D_{i j}$ is the squared Euclidean distance corresponding to $\mu_{i j}$. While the rest of the Lagrangian multipliers $\left\{\mu_{v w}\right\}=0$. Thus, from the second KKT condition in (23), we have $\mu_{i j}=1$. In this case, the matrix $\Psi^{\{1, i j\}}$ can be obtained by solving the equations that

$$
\left\{\begin{array}{l}
\varphi \Psi^{\{1, i j\}} \mathbf{u}_{i j} \mathbf{u}_{i j}^{T}-\lambda \Psi^{\{1, i j\}}=0 \\
\operatorname{Tr}\left(\Psi^{\{1, i j\}}\left(\Psi^{\{1, i j\}}\right)^{T}\right)-\frac{2(1-\varphi)}{\varphi}=0 .
\end{array}\right.
$$


Denote vector $\mathbf{u}_{i j}$ and matrix $\Psi^{\{1, i j\}}$ by

$$
\mathbf{u}_{i j}=\left[u_{i j, 1}, u_{i j, 2}\right]^{T} \quad \text { and } \quad \Psi^{\{1, i j\}}=\left[\psi_{1}^{\{1, i j\}}, \psi_{2}^{\{1, i j\}}\right]
$$

respectively, where the $2 \times 1$ vector $\psi_{a}^{\{1, i j\}}$ is the $a$-th column of matrix $\Psi^{\{1, i j\}}$ for $a=1,2$. After some manipulations, $\Psi^{\{1, i j\}}$ can be represented by

$$
\underbrace{\left[\begin{array}{cc}
\varphi\left(u_{i j, 1}\right)^{2}-\lambda & \varphi u_{i j, 1} u_{i j, 2} \\
\varphi u_{i j, 1} u_{2}^{\{1, i j\}} & \varphi\left(u_{i j, 2}\right)^{2}-\lambda
\end{array}\right]}_{\mathbf{F}_{m}}\left[\begin{array}{l}
\psi_{1}^{\{1, i j\}} \\
\psi_{2}^{\{1, i j\}}
\end{array}\right]^{T}=\mathbf{0}_{2 \times 1} .
$$

If $\operatorname{det}\left(\mathbf{F}_{i j}\right)=0$, i.e., $\lambda=\varphi\left(\left(u_{i j, 1}\right)^{2}+\left(u_{i j, 2}\right)^{2}\right)$, and $u_{i j, 1}, u_{i j, 2} \neq 0$, we have $\psi_{2}^{\{1, i j\}}=\frac{u_{i j, 2}}{u_{i j, 1}} \psi_{1}^{\{1, i j\}}$. Introducing two slack variables $\kappa_{i j, 1}$ and $\kappa_{i j, 2}$ for $\kappa_{i j, 1}, \kappa_{i j, 2} \in \mathbb{R}^{+}$, then matrix $\Psi^{\{1, i j\}}$ is given by (26a). Since $\operatorname{Tr}\left(\Psi^{\{1, i j\}}\left(\Psi^{\{1, i j\}}\right)^{T}\right)=\frac{2(1-\varphi)}{\varphi}$, the slack variables $\kappa_{i j, 1}$ and $\kappa_{i j, 2}$ should satisfy the constraint shown in (27).

Specially, when $\operatorname{det}\left(\mathbf{F}_{i j}\right)=0, u_{i j, 1}=u_{i j, 2}=0, \Psi^{\{1, i j\}}$ could be an arbitrary matrix that satisfies the relay transmission power constraint. Hence, the classical ANC with $\Psi^{\{1, i j\}}=\mathbf{H}_{2} \mathbf{I}_{2}$ could be one of the solution of the max-min problem. Else, if $\operatorname{det}\left(\mathbf{F}_{i j}\right) \neq 0$, there is no solution of $\psi_{1}^{\{1, i j\}}, \psi_{2}^{\{1, i j\}}$ to the function in (43).

In the second case when two Euclidean distances are equal to $D_{\text {min }}$, i.e., $\mu_{i j}>0, \mu_{v w}>0,\left\{\mu_{l z}\right\}=0, \lambda \neq 0$ for $i, j, v, w, l, z \in$ $\left\{1,2, \cdots, M^{2}\right\}, i \neq j, v \neq w, l \neq z$, from the complementary slackness conditions in (24), we have $D_{\min }^{2, i j}=D_{i j}=D_{v w}$, i.e.,

$$
D_{i j}^{\text {direct }}+\varphi\left\|\Psi^{\{2, i j\}} \mathbf{u}_{i j}\right\|_{2}^{2}=D_{v w}^{\text {direct }}+\varphi\left\|\Psi^{\{2, v w\}} \mathbf{u}_{v w}\right\|_{2}^{2} .
$$

After applying the rules $\|\mathbf{A u}\|_{2}^{2}=\operatorname{Tr}\left(\mathbf{A}^{T} \mathbf{A} \mathbf{u} \mathbf{u}^{T}\right)$ and $\operatorname{Tr}(\mathbf{A})+$ $\operatorname{Tr}(\mathbf{B})=\operatorname{Tr}(\mathbf{A}+\mathbf{B}),(44)$ can be further written as

$\operatorname{Tr}\left(\left(\Psi^{\{2, i j\}}\right)^{T} \Psi^{\{2, i j\}}\left(\mathbf{u}_{i j} \mathbf{u}_{i j}^{T}-\mathbf{u}_{i j} \mathbf{u}_{v w}^{T}\right)\right)=\frac{D_{v w}^{\text {direct }}-D_{i j}^{\text {direct }}}{\varphi}$.

Denote $\mathbf{B}^{\{i j\}}=\left[b_{11}^{\{i j\}}, b_{12}^{\{i j\}} ; b_{21}^{\{i j\}}, b_{22}^{\{i j\}}\right]=\left(\Psi^{\{2, i j\}}\right)^{T} \Psi^{\{2, i j\}}$ and

$$
\mathbf{U}^{\{i j\}}=\left[u_{11}^{\{i j\}}, u_{12}^{\{i j\}} ; u_{21}^{\{i j\}}, u_{22}^{\{i j\}}\right]=\mathbf{u}_{i j} \mathbf{u}_{i j}^{T}-\mathbf{u}_{v w} \mathbf{u}_{v w}^{T} .
$$

Then, combining with the second complementary slackness condition, we can obtain the intermediate variable $\mathbf{B}^{\{i j\}}$ by solving the following equations

$$
\begin{aligned}
& \operatorname{Tr}\left(\mathbf{B}^{\{i j\}} \mathbf{U}^{\{i j\}}\right)= u_{11}^{\{i j\}} b_{11}^{\{i j\}}+u_{21}^{\{i j\}} b_{12}^{\{i j\}}+u_{12}^{\{i j\}} b_{21}^{\{i j\}} \\
&+u_{22}^{\{i j\}} b_{22}^{\{i j\}}=\frac{D_{v w}^{\text {direct }}-D_{i j}^{\text {direct }}}{\varphi}, \\
& \operatorname{Tr}\left(\mathbf{B}^{\{i j\}}\right)=b_{11}^{\{i j\}}+b_{22}^{\{i j\}}=\frac{2(1-\varphi)}{\varphi} .
\end{aligned}
$$

In addition, we assume that matrix $\mathbf{B}^{\{i j\}}$ is a real symmetric matrix with equivalent diagonal elements, i.e., $b_{11}^{\{i j\}}=b_{22}^{\{i j\}}$ and $b_{12}^{\{i j\}}=b_{21}^{\{i j\}}$. With such assumption, on the one hand, we can obtain the deterministic solution of $\mathbf{B}^{\{i j\}}$ without introducing any slack variables. On the other hand, with the eigenvalue decomposition, matrix $\Psi^{\{2, i j\}}$ can be expressed by the eigenvectors and eigenvalues of matrix $\mathbf{B}^{\{i j\}}$. Specifically, we first derive matrix $\mathbf{B}^{\{i j\}}$ with (47) and the assumption that it is real and symmetric, which is shown in (48), shown at the bottom of the page.

For the real symmetric matrix $\mathbf{B}^{\{i j\}}$, the eigenvectors can be chosen such that they are real, orthogonal to each other and have norm one, that is

$$
\mathbf{B}^{\{i j\}}=\mathbf{Q}^{\{i j\}} \Lambda^{\{i j\}}\left(\mathbf{Q}^{\{i j\}}\right)^{T},
$$

where $\mathbf{Q}^{\{i j\}}$ is an orthogonal matrix, and $\Lambda^{\{i j\}}$ is a diagonal matrix whose entries are the eigenvalues of $\mathbf{B}^{\{i j\}}$. Since $\mathbf{B}^{\{i j\}}=\left(\Psi^{\{2, i j\}}\right)^{T} \Psi^{\{2, i j\}}, \mathbf{B}^{\{i j\}}$ is a positive definite matrix with positive eigenvalues. If there does not exist positive solution of $\mathbf{B}^{\{i j\}}$ in (48), we cannot find a solution for $\Psi^{\{2, i j\}}$. Otherwise, we have matrix $\Psi^{\{2, i j\}}=\mathbf{Q}^{\{i j\}}\left|\Lambda^{\{i j\}}\right|^{\frac{1}{2}}$ as given by (26b). ${ }^{2}$

\section{B. Proof of Theorem 2}

In the first case, when only one squared Euclidean distance is equal to $D_{\min }$, i.e., $\mu_{i j}=1,\left\{\mu_{v w}\right\}=0$ for $i, j, v, w \in$ $\left\{1,2, \cdots, M^{2}\right\}, i \neq j, v \neq w$ and $\lambda \neq 0$, from (36b), we have

$$
\begin{aligned}
& \mathbf{C}_{1} \mathbf{E}_{S} \widetilde{\mathbf{d}}_{i j}^{\Re}\left(\widetilde{\mathbf{d}}_{i j}^{\Re}\right)^{T}+\mathbf{C}_{2} \mathbf{E}_{S} \widetilde{\mathbf{d}}_{i j}^{\mathfrak{\Im}}\left(\widetilde{\mathbf{d}}_{i j}^{\Im}\right)^{T}+\mathbf{C}_{3} \mathbf{E}_{S} \widetilde{\mathbf{d}}_{i j}^{\Re}\left(\widetilde{\mathbf{d}}_{i j}^{\Im}\right)^{T} \\
& -\lambda \mathbf{E}_{\mathcal{S}}=\mathbf{0}_{2 \times 2} \text {, }
\end{aligned}
$$

where $\mathbf{C}_{1}=\mathbf{H}_{1} \mathbf{H}_{1}^{T}+\varphi\left(\Psi \mathbf{H}_{\mathcal{R}}\right)\left(\Psi \mathbf{H}_{\mathcal{R}}\right)^{T}, \mathbf{C}_{2}=\left(\mathbf{I}_{2^{\prime}} \mathbf{H}_{1}\right)\left(\mathbf{I}_{2^{\prime}} \mathbf{H}_{1}\right)^{T}+$ $\varphi\left(\Psi \mathbf{I}_{2^{\prime}} \mathbf{H}_{\mathcal{R}}\right)\left(\Psi \mathbf{I}_{2^{\prime}} \mathbf{H}_{\mathcal{R}}\right)^{T}$, and $\mathbf{C}_{3}=2\left(\left(\mathbf{I}_{2^{\prime}} \mathbf{H}_{1}\right)^{T} \mathbf{H}_{1}+\varphi\left(\Psi \mathbf{I}_{2^{\prime}} \mathbf{H}_{\mathcal{R}}\right)^{T}\right.$ $\left.\Psi \mathbf{H}_{\mathcal{R}}\right)$. Denote $\mathbf{E}_{\mathcal{S}}=\operatorname{Diag}\left(\sqrt{E_{\text {total }} \cos \left(\vartheta_{i j}\right)}, \sqrt{E_{\text {total }} \sin \left(\vartheta_{i j}\right)}\right)$

\footnotetext{
${ }^{2}$ Here, we apply the absolute value on matrix $\Lambda^{\{i j\}}$ because we can always set the eigenvalues of $\mathbf{B}^{\{i j\}}$ as $\max \left\{b_{11}^{\{i j\}}, b_{12}^{\{i j\}}\right\}-\min \left\{b_{11}^{\{i j\}}, b_{12}^{\{i j\}}\right\}$ and $\max \left\{b_{11}^{\{i j\}}, b_{12}^{\{i j\}}\right\}+\min \left\{b_{11}^{\{i j\}}, b_{12}^{\{i j\}}\right\}$, which will guarantee that $\Psi^{\{2, i j\}}$ is a real matrix.
}

$$
\mathbf{B}^{\{i j\}}=\left[\begin{array}{cc}
\frac{1-\varphi}{\varphi} & \frac{\left(D_{v w}^{\mathrm{direct}}-D_{i j}^{\mathrm{direct}}\right)-2(1-\varphi)\left(u_{11}^{\{i j\}}+u_{22}^{\{i j\}}\right)}{\varphi\left(u_{12}^{\{i j\}}+u_{21}^{\{i j\}}\right)} \\
\frac{\left(D_{v w}^{\mathrm{direct}}-D_{i j}^{\mathrm{direct}}\right)-2(1-\varphi)\left(u_{11}^{\{i j\}}+u_{22}^{\{i j\}}\right)}{\varphi\left(u_{12}^{\{i j\}}+u_{21}^{\{i j\}}\right)} & \frac{1-\varphi}{\varphi}
\end{array}\right]
$$


for $\vartheta_{i j} \in(0, \pi / 2)$. Expressing the matrices $\mathbf{C}_{q}$ by its elements as $\left[c_{q}^{11}, c_{q}^{12} ; c_{q}^{21}, c_{q}^{22}\right]$ for $q=1,2,3$. Define $\widetilde{d}_{i j}^{\Re}(p)$ as the $p$-th element of the vector $\tilde{d}_{i j}^{\Re(}(p)$ for $p=1,2$. Same definition can be applied to $\widetilde{d}_{i j}^{\Im}$. Hence, the left hand side of (50) can be rewritten as given in (51), shown at the bottom of the page, where we have (52), also shown at the bottom of the page, and

$$
\begin{aligned}
& \eta_{1, i j}=\left(\widetilde{d}_{i j}^{\Re}(1)\right)^{2} c_{1}^{11}+\left(\widetilde{d}_{i j}^{\Im}(1)\right)^{2} c_{2}^{11}+\widetilde{d}_{i j}^{\Re}(1) \widetilde{d}_{i j}^{\Im}(1) c_{3}^{11}, \\
& \eta_{2, i j}=\widetilde{d}_{i j}^{\Re}(1) \widetilde{d}_{i j}^{\Re}(2) c_{1}^{12}+\widetilde{d}_{i j}^{\Im}(1) \widetilde{d}_{i j}^{\Im}(2) c_{2}^{12}+\widetilde{d}_{i j}^{\Im}(1) \widetilde{d}_{i j}^{\Re}(2) c_{3}^{12} \text {, } \\
& \eta_{3, i j}=\widetilde{d}_{i j}^{\Re}(1) \widetilde{d}_{i j}^{\Re}(2) c_{1}^{11}+\widetilde{d}_{i j}^{\Im}(1) \tilde{d}_{i j}^{\Im}(2) c_{2}^{11}+\widetilde{d}_{i j}^{\Im}(2) \widetilde{d}_{i j}^{\Re}(1) c_{3}^{11} \text {, } \\
& \eta_{4, i j}=\left(\widetilde{d}_{i j}^{\Re}(2)\right)^{2} c_{1}^{12}+\left(\tilde{d}_{i j}^{\Im}(2)\right)^{2} c_{2}^{12}+\tilde{d}_{i j}^{\Im}(2) \widetilde{d}_{i j}^{\Re}(2) c_{3}^{12}, \\
& \eta_{5, i j}=\left(\widetilde{d}_{i j}^{\Re}(1)\right)^{2} c_{1}^{21}+\left(\widetilde{d}_{i j}^{\Im}(1)\right)^{2} c_{2}^{21}+\widetilde{d}_{i j}^{\Im}(1) \widetilde{d}_{i j}^{\Re}(1) c_{3}^{21}, \\
& \eta_{6, i j}=\widetilde{d}_{i j}^{\Re}(1) \widetilde{d}_{i j}^{\Re}(2) c_{1}^{22}+\tilde{d}_{i j}^{\Im}(1) \widetilde{d}_{i j}^{\Im}(2) c_{2}^{22}+\tilde{d}_{i j}^{\Im}(1) \widetilde{d}_{i j}^{\Re}(2) c_{3}^{22} \text {, } \\
& \eta_{7, i j}=\widetilde{d}_{i j}^{\Re}(1) \widetilde{d}_{i j}^{\Re}(2) c_{1}^{21}+\tilde{d}_{i j}^{\Im}(1) \tilde{d}_{i j}^{\Im}(2) c_{2}^{21}+\widetilde{d}_{i j}^{\Im}(2) \widetilde{d}_{i j}^{\Re}(1) c_{3}^{21}, \\
& \eta_{8, i j}=\left(\widetilde{d}_{i j}^{\Re}(2)\right)^{2} c_{1}^{22}+\left(\widetilde{d}_{i j}^{\Im}(2)\right)^{2} c_{2}^{22}+\widetilde{d}_{i j}^{\Im}(2) \widetilde{d}_{i j}^{\Re}(2) c_{3}^{22} \text {, }
\end{aligned}
$$

and it is equivalent to

$$
\underbrace{\left[\begin{array}{cc}
\left(\eta_{1, i j}-\lambda\right) & \eta_{2, i j} \\
\eta_{3, i j} & \eta_{4, i j} \\
\eta_{5, i j} & \eta_{6, i j} \\
\eta_{7, i j} & \left(\eta_{8, i j}-\lambda\right)
\end{array}\right]}_{\mathbf{G}_{i j}}\left[\begin{array}{l}
\sqrt{E_{\text {total }} \sin (\vartheta)} \\
\sqrt{E_{\text {total }} \cos (\vartheta)}
\end{array}\right]=\mathbf{0}_{4 \times 1} .
$$

If $\operatorname{rank}\left(\mathbf{G}_{i j}\right)=2$, then $\mathbf{E}_{\mathcal{S}}=\mathbf{0}_{2 \times 2}$. Otherwise, if $\operatorname{rank}\left(\mathbf{G}_{i j}\right)=$ 1, i.e., the column vectors of $\mathbf{G}_{i j}$ are linearly dependent, we can express $\vartheta$ as $\vartheta=\arctan \left(-\frac{\eta_{4, i j}}{\eta_{3, i j}}\right)$. Then the optimal $\mathbf{E}_{\mathcal{S}}$ is given by (39a). In the second case, when two squared Euclidean distances are equal to $D_{\min }$, i.e., $\mu_{i j}>0, \mu_{v w}>0,\left\{\mu_{l z}\right\}=0$, $\lambda \neq 0$, from the slack condition (37a), we have

$$
\begin{aligned}
& \left\|\mathbf{H}_{1} \mathbf{E}_{\mathcal{S}} \widetilde{\mathbf{d}}_{i j}^{\Re}+\mathbf{I}_{2^{\prime}} \mathbf{H}_{1} \mathbf{E}_{\mathcal{S}} \widetilde{\mathbf{d}}_{i j}^{\mathfrak{S}}\right\|_{2}^{2} \\
& +\varphi\left\|\Psi \mathbf{H}_{\mathcal{R}} \mathbf{E}_{\mathcal{S}} \widetilde{\mathbf{d}}_{i j}^{\Re}+\Psi \mathbf{I}_{2^{\prime}} \mathbf{H}_{\mathcal{R}} \mathbf{E}_{S} \widetilde{\mathbf{d}}_{i j}^{\mathfrak{S}}\right\|_{2}^{2} \\
& =\left\|\mathbf{H}_{1} \mathbf{E}_{\mathcal{S}} \widetilde{\mathbf{d}}_{v w}^{\Re}+\mathbf{I}_{2^{\prime}} \mathbf{H}_{1} \mathbf{E}_{S} \widetilde{\mathbf{d}}_{v w}^{\mathfrak{I}}\right\|_{2}^{2} \\
& \quad+\varphi\left\|\Psi \mathbf{H}_{\mathcal{R}} \mathbf{E}_{\mathcal{S}} \widetilde{\mathbf{d}}_{v w}^{\Re}+\Psi \mathbf{I}_{2^{\prime}} \mathbf{H}_{\mathcal{R}} \mathbf{E}_{\mathcal{S}} \widetilde{\mathbf{d}}_{v w}^{\mathfrak{S}}\right\|_{2}^{2},
\end{aligned}
$$

which is equivalent to

$$
\begin{aligned}
& \left(\widetilde{\mathbf{d}}_{i j}^{\Re}\right)^{T} \mathbf{E}_{\mathcal{S}}^{T} \mathbf{L}_{1} \mathbf{E}_{\mathcal{S}} \widetilde{\mathbf{d}}_{i j}^{\Re}+\left(\widetilde{\mathbf{d}}_{i j}^{\mathfrak{S}}\right)^{T} \mathbf{E}_{\mathcal{S}}^{T} \mathbf{L}_{2} \mathbf{E}_{\mathcal{S}} \widetilde{\mathbf{d}}_{\mathrm{ij}}^{\mathfrak{S}} \\
& +2\left(\widetilde{\mathbf{d}}_{i j}^{\Im}\right)^{T} \mathbf{E}_{\mathcal{S}}^{T} \mathbf{L}_{3} \mathbf{E}_{\mathcal{S}} \widetilde{\mathbf{d}}_{i j}^{\Re} \\
& =\left(\widetilde{\mathbf{d}}_{v w}^{\Re}\right)^{T} \mathbf{E}_{\mathcal{S}}^{T} \mathbf{L}_{1} \mathbf{E}_{\mathcal{S}} \widetilde{\mathbf{d}}_{v w}^{\Re}+\left(\widetilde{\mathbf{d}}_{v w}^{\mathfrak{I}}\right)^{T} \mathbf{E}_{\mathcal{S}}^{T} \mathbf{L}_{2} \mathbf{E}_{\mathcal{S}} \widetilde{\mathbf{d}}_{v w}^{\mathfrak{I}} \\
& +2\left(\widetilde{\mathbf{d}}_{v w}^{\mathfrak{S}}\right)^{T} \mathbf{E}_{\mathcal{S}}^{T} \mathbf{L}_{3} \mathbf{E}_{\mathcal{S}} \widetilde{\mathbf{d}}_{v w}^{\Re},
\end{aligned}
$$

where $\mathbf{L}_{1}=\mathbf{H}_{1}^{T} \mathbf{H}_{1}+\varphi \mathbf{H}_{\mathcal{R}}^{T} \Psi^{T} \Psi \mathbf{H}_{\mathcal{R}}, \mathbf{L}_{2}=\mathbf{H}_{1}^{T}\left(\mathbf{I}_{2}^{\prime}\right)^{T} \mathbf{I}_{2}^{\prime} \mathbf{H}_{1}+$ $\varphi \mathbf{H}_{\mathcal{R}}^{T}\left(\mathbf{I}_{2}^{\prime}\right)^{T} \Psi^{T} \Psi \mathbf{I}_{2}^{\prime} \mathbf{H}_{\mathcal{R}}$, and $\mathbf{L}_{3}=\mathbf{H}_{1}^{T}\left(\mathbf{I}_{2}^{\prime}\right)^{T} \mathbf{H}_{1}+\varphi \mathbf{H}_{\mathcal{R}}^{T}\left(\mathbf{I}_{2}^{\prime}\right)^{T} \Psi^{T} \Psi \mathbf{H}_{\mathcal{R}}$. Expressing the matrices $\mathbf{L}_{q}$ by its elements as $\left[l_{q}^{11}, l_{q}^{12} ; l_{q}^{21}, l_{q}^{22}\right]$ for $q=1,2,3$. Hence, (56) can be further written as in (52). It is equivalent to

$$
\underbrace{\left[\begin{array}{ll}
\left(\varphi_{1, i j}-\varphi_{1, v w}\right) & \left(\varphi_{2, i j}-\varphi_{2, v w}\right) \\
\left(\varphi_{3, i j}-\varphi_{3, v w}\right) & \left(\varphi_{4, i j}-\varphi_{4, v w}\right) \\
\left(\varphi_{5, i j}-\varphi_{5, v w}\right) & \left(\varphi_{6, i j}-\varphi_{6, v w}\right) \\
\left(\varphi_{7, i j}-\varphi_{7, v w}\right) & \left(\varphi_{8, i j}-\varphi_{8, v w}\right)
\end{array}\right]}_{\mathbf{Z}_{i j}}\left[\begin{array}{l}
E_{\text {total }} \sqrt{\sin (\vartheta)} \\
E_{\text {total }} \sqrt{\cos (\vartheta)}
\end{array}\right]=0,
$$

where

$$
\begin{aligned}
& \varphi_{1, m t}=l_{1}^{11}\left(\widetilde{d}_{m t}^{\Re \mathfrak{\Re}}(1)\right)^{2}+l_{2}^{11}\left(\widetilde{d}_{m t}^{\mathfrak{S}}(1)\right)^{2} \\
& +2 l_{3}^{11} \widetilde{d}_{m t}^{\Re}(1) \tilde{d}_{m t}^{\mathfrak{S}}(1), \\
& \varphi_{2, m t}=l_{1}^{21} \widetilde{d}_{m t}^{\Re}(1) \widetilde{d}_{m t}^{\Re}(2)+l_{2}^{21} \widetilde{d}_{m t}^{\mathfrak{S}}(1) \widetilde{d}_{m t}^{\mathfrak{I}}(2) \\
& +2 l_{3}^{21} \widetilde{d}_{m t}^{\Re}(1) \tilde{d}_{m t}^{\mathfrak{S}}(2), \\
& \varphi_{3, m t}=l_{1}^{12}\left(\widetilde{d}_{m t}^{\Re}(1)\right)^{2}+l_{2}^{12}\left(\widetilde{d}_{m t}^{\Im}(1)\right)^{2} \\
& +2 l_{3}^{12} \widetilde{d}_{m t}^{\Re}(1) \widetilde{d}_{m t}^{\mathfrak{S}}(1), \\
& \varphi_{4, m t}=l_{1}^{22} \widetilde{d}_{m t}^{\Re}(1) \widetilde{d}_{m t}^{\Re}(2)+l_{2}^{22} \widetilde{d}_{m t}^{\mathfrak{S}}(1) \widetilde{d}_{m t}^{\mathfrak{S}}(2) \\
& +2 l_{3}^{22} \widetilde{d}_{m t}^{\Re}(1) \widetilde{d}_{m t}^{\Im}(2) \text {, } \\
& \varphi_{5, m t}=l_{1}^{11} \widetilde{d}_{m t}^{\Re}(1) \widetilde{d}_{m t}^{\Re}(2)+l_{2}^{11} \widetilde{d}_{m t}^{\Im}(1) \widetilde{d}_{m t}^{\Im}(2) \\
& +2 l_{3}^{11} \widetilde{d}_{m t}^{\Re}(2) \widetilde{d}_{m t}^{\Im}(1), \\
& \varphi_{6, m t}=l_{1}^{21}\left(\widetilde{d}_{m t}^{\Re}(2)\right)^{2}+l_{2}^{21}\left(\widetilde{d}_{m t}^{\mathfrak{S}}(2)\right)^{2} \\
& +2 l_{3}^{21} \widetilde{d}_{m t}^{\Re}(2) \tilde{d}_{m t}^{\mathfrak{S}}(2), \\
& \varphi_{7, m t}=l_{1}^{12} \widetilde{d}_{m t}^{\Re}(1) \widetilde{d}_{m t}^{\Re}(2)+l_{2}^{12} \widetilde{d}_{m t}^{\Im}(1) \widetilde{d}_{m t}^{\Im}(2) \\
& +2 l_{3}^{12} \widetilde{d}_{m t}^{\Re}(2) \widetilde{d}_{m t}^{\mathfrak{S}}(1), \\
& \varphi_{8, m t}=l_{1}^{22}\left(\widetilde{d}_{m t}^{\Re}(2)\right)^{2}+l_{2}^{22}\left(\tilde{d}_{m t}^{\mathfrak{S}}(2)\right)^{2} \\
& +2 l_{3}^{22} \widetilde{d}_{m t}^{\Re}(2) \widetilde{d}_{m t}^{\mathfrak{S}}(2),
\end{aligned}
$$$$
m t \in\{i j, v w\}
$$

$$
\left[\begin{array}{cc}
\left(\eta_{1, i j}-\lambda\right) \sqrt{E_{\text {total }} \sin (\vartheta)}+\eta_{2, i j} \sqrt{E_{\text {total }} \cos (\vartheta)} & \eta_{3, i j} \sqrt{E_{\text {total }} \sin (\vartheta)}+\eta_{4, i j} \sqrt{E_{\text {total }} \cos (\vartheta)} \\
\eta_{5, i j} \sqrt{E_{\text {total }} \sin (\vartheta)}+\eta_{6, i j} \sqrt{E_{\text {total }} \cos (\vartheta)} & \eta_{7, i j} \sqrt{E_{\text {total }} \sin (\vartheta)}+\left(\eta_{8, i j}-\lambda\right) \sqrt{E_{\text {total }} \cos (\vartheta)}
\end{array}\right]
$$

$$
\left[\begin{array}{cc}
\left(\varphi_{1, i j}-\varphi_{1, v w}\right) E_{1}^{2}+\left(\varphi_{2, i j}-\varphi_{2 p}\right) E_{1} E_{2} & \left(\varphi_{3, i j}-\varphi_{3, v w}\right) E_{1} E_{2}+\left(\varphi_{4, i j}-\varphi_{4, v w}\right) E_{2}^{2} \\
\left(\varphi_{5, i j}-\varphi_{5, v w}\right) E_{1}^{2}+\left(\varphi_{6, i j}-\varphi_{6, v w}\right) E_{1} E_{2} & \left(\varphi_{7, i j}-\varphi_{7, v w}\right) E_{1} E_{2}+\left(\varphi_{8, i j}-\varphi_{8, v w}\right) E_{2}^{2}
\end{array}\right]=\mathbf{0}_{2 \times 2}
$$


If $\operatorname{rank}\left(\mathbf{Z}_{i j}\right)=2$, then $\mathbf{E}_{\mathcal{S}}=\mathbf{0}_{2 \times 2}$. Otherwise, if $\operatorname{rank}\left(\mathbf{Z}_{i j}\right)=1$, i.e., the column vectors of $\mathbf{Z}_{i j}$ are linearly dependent, we have $\vartheta=\arctan \left(-\frac{\varphi_{2, i j}-\varphi_{2, v w}}{\varphi_{1, i j}-\varphi_{1, v w}}\right)$. The optimal $\mathbf{E}_{\mathcal{S}}$ is given by (39b).

Note that the matrix $\mathbf{E}_{\mathcal{S}}$ has no solution in some of the cases for the strict requirements on the determinant of matrix $\mathbf{G}_{i j}$ defined in (54). In this case, we assume that $\frac{1}{\phi} \leq \tan (\vartheta) \leq \phi$ for $\phi>1$. When there is no solution satisfying the KKT conditions, we substitute the boundary values of $\vartheta$ in matrix $\mathbf{E}_{\mathcal{S}}$ and the one which maximizes $D_{\min }$ is kept as the 'optimal' value.

\section{REFERENCES}

[1] T. Cover and A. Gamal, "Capacity theorems for the relay channel," IEEE Trans. Inform. Theory, vol. 25, no. 5, pp. 572-584, Sep. 1979.

[2] J. N. Laneman, D. N. C. Tse, and G. W. Wornell, "Cooperative diversity in wireless networks: Efficient protocols and outage behavior," IEEE Trans. Inform. Theory, vol. 50, no. 12, pp. 3062-3080, Dec. 2004.

[3] R. Ahlswede, N. Cai, S. Y. R. Li, and R. Yeung, "Network information flow," IEEE Trans. Inform. Theory, vol. 46, no. 4, pp. 1204-1216, Jul. 2000.

[4] S. Y. R. Li, R. W. Yeung, and N. Cai, "Linear network coding," IEEE Trans. Inform. Theory, vol. 49, no. 2, pp. 371-381, Feb. 2003.

[5] S. Katti et al., "Xors in the air: Practical wireless network coding," in Proc. ACM SIGCOMM, Sep. 2006, pp. 243-254.

[6] G. Kramer, M. Gastpar, and P. Gupta, "Cooperative strategies and capacity theorems for relay networks," IEEE Trans. Inform. Theory, vol. 51, no. 9, pp. 3037-3063, Sep. 2005.

[7] X. Bao and J. Li, "Adaptive Network Coded Cooperation (ANCC) for wireless relay networks: Matching code-on-graph with network-ongraph," IEEE Trans. Wireless Commun., vol. 7, no. 2, pp. 574-583, Feb. 2008.

[8] J. Du, M. Xiao, and M. Skoglund, "Cooperative network coding strategies for wireless relay networks with backhaul," IEEE Trans. Commun., vol. 59, no. 9, pp. 2502-2514, Sep. 2011.

[9] M. Xiao and M. Skoglund, "Multiple-user cooperative communications based on linear network coding," IEEE Trans. Commun., vol. 58, no. 12, pp. 3345-3351, Dec. 2010.

[10] J. Li, J. Yuan, R. Malancy, M. Xiao, and W. Chen, "Full-diversity binary frame-wise network coding for multiple-source multiple-relay networks over slow-fading channels," IEEE Trans. Veh. Technol., vol. 61, no. 3, pp. 1346-1360, Mar. 2012.

[11] J. Li, J. Yuan, R. Malaney, M. Azmi, and M. Xiao, "Network coded LDPC code design for a multi-source relaying system," IEEE Trans. Wireless Commun., vol. 10, no. 5, pp. 1538-1551, May 2011.

[12] J. Li, W. Chen, Z. Lin, and B. Vucetic, "Design of physical layer network coded LDPC code for a multiple-access relaying system," IEEE Commun. Lett., vol. 17, no. 4, pp. 749-752, Apr. 2013.

[13] M. Xiao, J. Kliewer, and M. Skoglund, "Design of network codes for multiple-user multiple-relay wireless networks," IEEE Trans. Commun., vol. 60, no. 12, pp. 3755-3766, Dec. 2012.

[14] S. Zhang, S. C. Liew, and P. P. Lam, "Hot topic: Physical-layer network coding," in Proc. ACM MobiCom, 2006, pp. 358-365.

[15] V. T. Muralidharan and B. S. Rajan, "Physical layer network coding for the k-user multiple access relay channel," IEEE Trans. Wireless Commun., vol. 12, no. 6, pp. 3107-3119, Jun. 2013.

[16] B. Nazer and M. Gastpar, "Compute-and-forward: Harnessing interference through structured codes," IEEE Trans. Inform. Theory, vol. 57, no. 10, pp. 6463-6486, Oct. 2011.

[17] C. Feng, D. Silva, and F. R. Kschischang, "An algebraic approach to physical-layer network coding," IEEE Trans. Inform. Theory, vol. 59, no. 11, pp. 7576-7596, Nov. 2013.

[18] Q. T. Sun, J. Yuan, T. Huang, and W. K. Shum, "On lattice-partition based physical-layer network coding over GF(4),” IEEE Commun. Lett., vol. 61, no. 7, pp. 2713-2725, Jul. 2013.

[19] P. Popovski and H. Yomo, "Wireless network coding by amplify-and forward for bi-directional traffic flows," IEEE Commun. Lett., vol. 11, no. 1, pp. 16-18, Jan. 2007.

[20] S. Katti, S. Gollakota, and D. Katabi, "Embracing wireless interference: Analog network coding," in Proc. ACM SIGCOMM, Kyoto, Aug. 2007, pp. 397-408.

[21] A. Zhao, C. He, and L. G. Jiang, "Outage behavior in wireless networks with analog network coding," IEEE Trans. Veh. Technol., vol. 61, no. 7, pp. 3352-3360, Sep. 2012.

[22] A. Argyriou and A. Pandharipande, "Cooperative protocol for analog network coding in distributed wireless networks," IEEE Trans. Wireless Commun., vol. 9, no. 10, pp. 3112-3119, Oct. 2010.
[23] L. Song, G. Hong, B. Jiao, and M. Debbah, "Joint relay selection and analog network coding using differential modulation in two-way relay channels," IEEE Trans. Veh. Technol., vol. 59, no. 6, pp. 2932-2939, Jul. 2010.

[24] S. Zhang, S. C. Liew, Q. Zhou, L. Lu, and H. Wang, "Non-memoryless analog network coding in two-way relay channel," in Proc. IEEE ICC, Jun. 2011, pp. 1-6.

[25] W. Guan and K. J. R. Liu, "Diversity analysis of analog network coding with multi-user interferences," IEEE Trans. Wireless Commun., vol. 12, no. 2, pp. 668-679, Feb. 2013.

[26] S. Wei et al., "Power adaptive network coding for a non-orthogonal multiple-access relay channel," IEEE Trans. Commun., vol. 62, no. 3, pp. 872-887, Mar. 2014.

[27] B. Jiang, F. Gao, X. Gao, and A. Nallanathan, "Channel estimation and training design for two-way relay networks with power allocation," IEEE Trans. Wireless Commun., vol. 9, no. 6, pp. 2022-2032, Jun. 2010.

[28] Q. Zhao, Z. Zhou, J. Li, and B. Vucetic, "Joint semi-blind channel estimation and synchronization in two way relay networks," IEEE Trans. Veh. Technol., vol. 63, no. 7, pp. 3276-3293, Sep. 2014.

[29] C. P. Lee, S. C. Lin, H. J. Su, and H. V. Poor, "Multi-user lattice coding for the multiple-access relay channel," IEEE Trans. Inform. Theory, to be published.

[30] M. E. Soussi, A. Zaidi, and L. Vandendorpe, "Compute-and-forward on a multiaccess relay channel: Coding and symmetric-rate optimization," IEEE Trans. Wireless Commun., vol. 13, no. 4, pp. 1932-1947, Apr. 2014.

[31] S. Zhang and S. C. Liew, "Channel coding and decoding in a relay system operated with physical-layer network coding," IEEE J. Select Areas Commun., vol. 27, no. 5, pp. 788-796, Jun. 2009.

[32] P. Popovski and H. Yomo, "The anti-packets can increase the achievable throughput of a wireless multi-hop network," in Proc. IEEE ICC, Jun. 2006, vol. 9, pp. 3885-3890.

[33] T. Wang and G. B. Giannakis, "Complex field network coding for multiuser cooperative communications," IEEE J. Select. Areas Commun., vol. 26, no. 3, pp. 561-571, Apr. 2008.

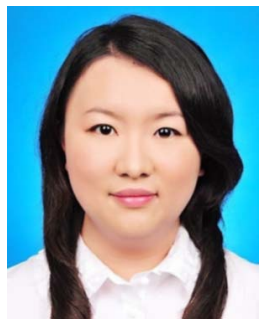

Sha Wei received the B.S. and M.S. degrees in electronic engineering from the University of Electronic Science and Technology of China (UESTC), Chengdu, and Shanghai Jiao Tong University, Shanghai, China, in 2007 and 2009, respectively, and the $\mathrm{Ph} . \mathrm{D}$. degree in electronic engineering from Shanghai Jiao Tong University, Shanghai, in 2014. Her current research interests include wireless network coding and cooperative communications.

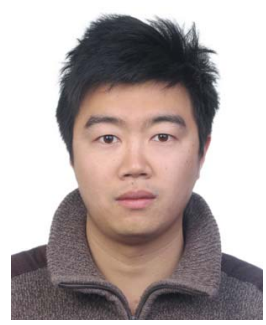

Jun Li (M'09) received the Ph.D. degree in electronic engineering from Shanghai Jiao Tong University, Shanghai, China, in 2009. From January 2009 to June 2009, he worked in the Department of Research and Innovation, Alcatel Lucent Shanghai Bell as a Research Scientist. From June 2009 to April 2012, he was a Postdoctoral Fellow at the School of Electrical Engineering and Telecommunications, the University of New South Wales, Sydney, N.S.W., Australia. From April 2012 to present he has been a Research Fellow at the School of Electrical Engineering, the University of Sydney, Sydney, N.S.W., Australia. He served as the Technical Program Committee member for several international conferences such as APCC2009, APCC2010, VTC2011 (Spring), ICC2011, TENCON2012, APCC2013, VTC2014 (Fall), and ICC2014. His research interests include network information theory, channel coding theory, wireless network coding and cooperative communications.

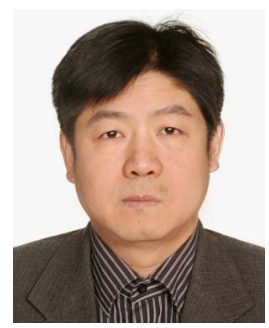

Wen Chen (M'03-SM'11) received the B.S. and M.S. degrees from Wuhan University, Wuhan, China, in 1990 and 1993, respectively, and the Ph.D. degree from the University of Electro-Communications, Tokyo, Japan, in 1999. He was a Researcher of Japan Society for the Promotion of Sciences (JSPS) from 1999 through 2001. In 2001, he joined the University of Alberta, Canada, starting as a Postdoctoral Fellow with the Information Research Laboratory and continuing as a Research Associate in the Department of Electrical and Computer Engineering. Since 2006, he has been a Full Professor in the Department of Electronic Engineering, Shanghai Jiao Tong University, Shanghai, China, where he is also the Director of the Institute for Signal Processing and Systems. His interests cover network coding, cooperative communications, cognitive radio, and MIMO-OFDM systems. 


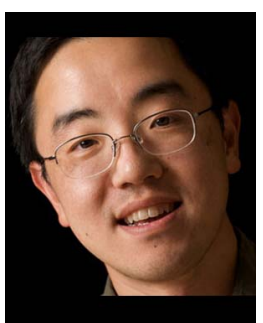

Lizhong Zheng received the B.S and M.S. degrees from the Department of Electronic Engineering, Tsinghua University, Beijing, China, in 1994 and 1997, respectively, and the Ph.D. degree from the Department of Electrical Engineering and Computer Sciences, University of California, Berkeley, CA, USA, in 2002. Since 2002, he has been working in the Department of Electrical Engineering and Computer Sciences, where he is currently a Professor of electrical engineering. His research interests include information theory, wireless networks, and statistical inference. He received Eli Jury award from UC Berkeley in 2002, IEEE INFORMATION THEORY SOCIETY PAPER AWARD in 2003, and NSF CAREER award in 2004, and the AFOSR Young Investigator Award in 2007.

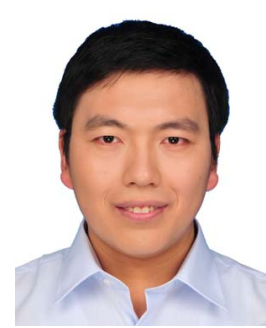

Hang Su received the B.S., M.S., and Ph.D. degrees in electronic engineering from Shanghai Jiao Tong University, Shanghai, China in 2007, 2009, and 2014, respectively. He is currently a PostDoc Fellow at School of Computer Science, Tsinghua University, Beijing, China. His current research interests include multimedia communication, computer vision and machine learning 